\title{
Efficacité du traitement endoscopique dans la prise en charge des fistules digestives anastomotiques après chirurgie pour cancer de l'œsophage
}

\section{T H È S E-A R T I C L E}

Présentée et publiquement soutenue devant

LA FACULTÉ DE MÉDECINE DE MARSEILLE

Le 19 Juin 2015

Par Madame Cécilia SERVAJEAN-DEFRANCHI

Née le 1er novembre 1986 à Paris (75)

Pour obtenir le grade de Docteur en Médecine

D.E.S. de GASTRO-ENTÉROLOGIE ET HÉPATOLOGIE

Membres du Jury de la Thèse :

Monsieur le Professeur GRIMAUD Jean-Charles

Président

Monsieur le Professeur BARTHET Marc

Assesseur

Monsieur le Professeur D' JOURNO Xavier Benoît

Assesseur

Monsieur le Professeur LEONE Marc

Assesseur

Monsieur le Docteur GASMI Mohamed

Directeur 



\section{Efficacité du traitement endoscopique dans la prise en charge des fistules digestives anastomotiques après chirurgie pour cancer de l'œsophage}

\section{T H È S E-A R T I C L E}

Présentée et publiquement soutenue devant

LA FACULTÉ DE MÉDECINE DE MARSEILLE

Le 19 Juin 2015

Par Madame Cécilia SERVAJEAN-DEFRANCHI

Née le 1er novembre 1986 à Paris (75)

Pour obtenir le grade de Docteur en Médecine

D.E.S. de GASTRO-ENTÉROLOGIE ET HÉPATOLOGIE

Membres du Jury de la Thèse :

Monsieur le Professeur GRIMAUD Jean-Charles

Président

Monsieur le Professeur BARTHET Marc

Assesseur

Monsieur le Professeur D' JOURNO Xavier Benoît

Assesseur

Monsieur le Professeur LEONE Marc

Assesseur

Monsieur le Docteur GASMI Mohamed

Directeur 


\title{
UNIVERSITE D'AIX-MARSEILLE
}

Président : Yvon BERLAND

\section{FACULTE DE MEDECINE}

\author{
Doyen : Georges LEONETTI
}

Vice-Doyen aux Affaires Générales : Patrick DESSI

Vice-Doyen aux Professions Paramédicales : Philippe BERBIS

Assesseurs :

* aux Etudes : Jean-Michel VITON

* à la Recherche : Jean-Louis MEGE

* aux Prospectives Hospitalo-Universitaires : Frédéric COLLART

* aux Enseignements Hospitaliers : Patrick VILLANI

* pour le Département Professionnel Continu : Fabrice BARLESI

* pour le Secteur Nord : Christian BRUNET

Chargés de mission :

* $1^{\text {er }}$ cycle : Jean-Marc DURAND et Marc BARTHET

* $2^{\text {ème }}$ cycle : Marie-Aleth RICHARD

* $3^{\text {eme }}$ cycle DES/DESC : Gilles BOUVENOT et Pierre-Edouard FOURNIER

* Licences-Masters-Doctorat : Pascal ADALIAN

* DU-DIU : Gérard SEBAHOUN

* Sciences Humaines et Sociales : Pierre LE COZ

* Préparation à l'ECN : Stéphane BERDAH

* Démographie Médicale et Filiarisation : Roland SAMBUC

* Relations Internationales : Philippe PAROLA

* DPC Spécialités Médicales : Gilbert HABIB

* DPC Spécialités Chirurgicales : Jean-Luc JOUVE

*DPC Médecine Générale : Yves FRANCES

* DPC Paramédicaux : Catherine METZLER/GUILLEMAIN

* Personnel BIATSS : Emmanuelle CHARAFFE-JAUFFRET

* Etudiants : Lola LOUSSERT

Chef des services généraux : $\quad *$ Pascale SOLO

Chefs de service :

* Communication : Ghislaine HANCY

* Examens : Marie-Thérèse ZAMMIT

* Scolarité Pédagogique : Christine GAUTHIER

* Maintenance : Philippe KOCK

* Intérieur : Joëlle FRAVEGA

\section{DOYENS HONORAIRES}

M. Yvon BERLAND

M. André ALI CHERIF

M. Jean-François PELLISSIER 


\section{PROFESSEURS HONORAIRES}

MM AGOSTINI Serge ALDIGHIERI René ALLIEZ Bernard AQUARON Robert ARGEME Maxime ASSADOURIAN Robert BAILLE Yves BARDOT André BERARD Pierre BERGOIN Maurice BERNARD Dominique BERNARD Pierre-Marie BERTRAND Edmond BISSET Jean-Pierre BLANC Bernard BONNEAU Henri BONNOIT Jean BORY Michel BOURGEADE Augustin BOUTIN Christian BOUVENOT Gilles BOUYALA Jean-Marie BREMOND Georges BRICOT René BUREAU Henri CAMBOULIVES Jean CANNONI Maurice CARCASSONNE Yves CARTOUZOU GuY CHAMLIAN Albert CHARREL Michel CHOUX Maurice CIANFARANI François CLEMENT Robert CODACCIONI Jean-Louis COMBALBERT André CORRIOL Jacques COTTE Gérard DALMAS Henri DESANTI Etienne DEVIN Robert DEVRED Philippe DJIANE Pierre DONNET Vincent DUCASSOU Jacques DUFOUR Michel FARISSE Jacques FARNARIER Georges FAVRE Roger FIECHI Marius FIGARELLA Jacques FRANCOIS Georges FUENTES Pierre GABRIEL Bernard GALINIER Louis GALLAIS Hervé GAMERRE Marc GARCIN Michel GASCARD Emile GAUTHIER André GERARD Raymond
MM GEROLAMI-SANTANDREA André GIUDICELLI Roger GIUDICELLI Sébastien GOUDARD Alain GOUIN François GRIMAUD Charles GRISOLI François GROULIER Pierre HADIDA/SAYAG Jacqueline HASSOUN Jacques HEIM Marc HOUEL Jean HUGUET Jean-François JAQUET Philippe JOUVE Paulette JUHAN Claude JUIN Pierre KAPHAN Gérard KASBARIAN Michel KHALIL Richard KLEISBAUER Jean-Pierre LACHARD Jean LAFFARGUE Pierre LEVY Samuel LOUCHET Edmond LOUIS René LUCIANI Jean-Marie MAGALON Guy MAGNAN Jacques MALLAN- MANCINI Josette MALMEJAC Claude MATTEI Jean François MERCIER Claude METGE Paul MICHOTEY Georges MILLET Yves MIRANDA François MONFORT Gérard MONGES André MONGIN Maurice MONTIES Jean-Raoul NICOLI René NOIRCLERC Michel OLMER Michel OREHEK Jean PAPY Jean-Jacques PAULIN Raymond PELLET William PELOUX Yves PENAUD Antony PENE Pierre PERRIMOND Henri PIANA Lucien PICAUD Robert PIGNOL Fernand POGGI Louis PONCET Michel POYEN Danièle PRIVAT Yvan QUILICHINI Francis RANQUE Jacques 
PROFESSEURS HONORAIRES

MM RANQUE Philippe

REBOUD Eugène

RICHAUD Christian

ROCHAT Hervé

ROHNER Jean-Jacques

ROUX Hubert

ROUX Michel

RUF Henri

RUFO Marcel

SAHEL José

SALAMON Georges

SALDUCCI Jacques

SAN MARCO Jean-Louis

SANKALE Marc

SARACCO Jacques

SARLES Henry

SARLES Jean-Claude

SCHIANO Alain

SCOTTO Jean-Claude

SEBAHOUN Gérard

SEITE Raymond

SERRATRICE Georges

SOULAYROL René

STAHL André

TAMALET Jacques

TOURNIGAND Pierre

TRIFAUD André

UNAL Daniel

VAGUE Philippe

VAGUE/JUHAN Irène

VANUXEM Paul

VERVLOET Daniel

VIGOUROUX Robert

WEILLER Pierre-Jean 


\section{8}

M. le Professeur

LEVY Samuel

$31 / 08 / 2011$

Mme le Professeur

JUHAN-VAGUE Irène

$31 / 08 / 2011$

PONCET Michel

$31 / 08 / 2011$

KASBARIAN Michel

$31 / 08 / 2011$

M. le Professeur

ROBERTOUX Pierre

$31 / 08 / 2011$

\section{9}

M. le Professeur

DJIANE Pierre

$31 / 08 / 2011$

M. le Professeur

VERVLOET Daniel

$31 / 08 / 2012$

2010

M. le Professeur

MAGNAN Jacques

$31 / 12 / 2014$

2011

M. le Professeur

DI MARINO Vincent

$31 / 08 / 2015$

MARTIN Pierre

$31 / 08 / 2015$

METRAS Dominique

$31 / 08 / 2015$

\section{2}

M. le Professeur

M. le Professeur

M. le Professeur

M. le Professeur

M. le Professeur

M. le Professeur

M. le Professeur

AUBANIAC Jean-Manuel

$31 / 08 / 2015$

BOUVENOT Gilles

$31 / 08 / 2015$

CAMBOULIVES Jean

$31 / 08 / 2015$

FAVRE Roger

$31 / 08 / 2015$

MATTEI Jean-François

$31 / 08 / 2015$

OLIVER Charles

$31 / 08 / 2015$

VERVLOET Daniel

$31 / 08 / 2015$

2013

M. le Professeur

BRANCHEREAU Alain

$31 / 08 / 2016$

CARAYON Pierre

$31 / 08 / 2016$

COZZONE Patrick

$31 / 08 / 2016$

DELMONT Jean

$31 / 08 / 2016$

HENRY Jean-François

$31 / 08 / 2016$

LE GUICHAOUA Marie-Roberte 31/08/2016

RUFO Marcel

$31 / 08 / 2016$

SEBAHOUN Gérard

$31 / 08 / 2016$

\section{4}

M. le Professeur

M. le Professeur

M. le Professeur

M. le Professeur

M. le Professeur

FUENTES Pierre

$31 / 08 / 2017$

GAMERRE Marc

$31 / 08 / 2017$

MAGALON Guy

$31 / 08 / 2017$

PERAGUT Jean-Claude

$31 / 08 / 2017$

WEILLER Pierre-Jean

$31 / 08 / 2017$ 


\section{PROFESSEURS DES UNIVERSITES-PRATICIENS HOSPITALIERS}

AGOSTINI Aubert

ALBANESE Jacques

ALESSANDRINI Pierre Surnombre

ALIMI Yves

AMABILE Philippe

AMBROSI Pierre

ARGENSON Jean-Noël

ASTOUL Philippe

ATTARIAN Shahram

AUDOUIN Bertrand

AUFFRAY Jean-Pierre Surnombre

AUQUIER Pascal

AUTILLO/TOUATI Amapola

AVIERINOS Jean-François

AZORIN Jean-Michel

AZULAY Jean-Philippe

BAILLY Daniel

BARDOT Jacques

BARLESI Fabrice

BARLIER-SETTI Anne

BARTHET Marc

BARTOLI Jean-Michel

BARTOLI Michel

BARTOLIN Robert

BARTOLOMEI Fabrice

BASTIDE Cyrille

BENSOUSSAN Laurent

BERBIS Philippe

BERDAH Stéphane

BERLAND Yvon

BERNARD Jean-Louis

BERNARD Jean-Paul

BEROUD Christophe

BERTUCCI François

BLADOU Franck

BLAISE Didier

BLANC Jean-Louis Surnombre

BLIN Olivier

BOLLINI Gérard Surnombre

BONGRAND Pierre

BONIN/GUILLAUME Sylvie

BONNET Jean-Louis

BOTTA Alain Surnombre

BOTTA/FRIDLUND Danielle

BOUBLI Léon

BRETELLE Florence

BROUQUI Philippe

BRUDER Nicolas

BRUE Thierry

BRUNET Christian

BRUNET Philippe

BURTEY Stéphane

CASANOVA Dominique

CAU Pierre Surnombre

CECCALDI Mathieu

CHABOT Jean-Michel

CHAGNAUD Christophe

CHAMBOST Hervé

CHAMPSAUR Pierre

CHANEZ Pascal

CHARAFFE-JAUFFRET Emmanuelle
CHIARONI Jacques

CHARPIN Denis

CHAUMOITRE Kathia

CHAUVEL Patrick Surnombre

CHINOT Olivier

CHOSSEGROS Cyrille

CLAVERIE Jean-Michel

COLLART Frédéric

CONTE-DEVOLX Bernard Surnombre

COSTELLO Régis

COULANGE Christian Surnombre

COWEN Didier

CRAVELLO Ludovic

CUISSET Thomas

CURVALE Georges

DA FONSECA David

DANIEL Laurent

DARMON Patrice

D'ERCOLE Claude

D'JOURNO Xavier

DE LAGAUSIE Pascal

DE MICCO Philippe Surnombre

DEHARO Jean-Claude

DELARQUE Alain

DELPERO Jean-Robert

DENIS Danièle

DESSEIN Alain

DESSI Patrick

DISDIER Patrick

DODDOLI Christophe

DRANCOURT Michel

DUBUS Jean-Christophe

DUFFAUD Florence

DUFOUR Henry

DURAND Jean-Marc

DUSSOL Bertrand

ENJALBERT Alain

FAUGERE Gérard

FELICIAN Olvier

FENOLLAR Florence

FIGARELLA/BRANGER Dominique

FLECHER Xavier

FONTES Michel

FOURNIER Pierre-Edouard

FRAISSE Alain

FRANCES Yves

FUENTES Stéphane

GABERT Jean

GAINNIER Marc

GARCIA Stéphane

GARIBOLDI Vlad

GARNIER Jean-Marc Surnombre

GENTILE Stéphanie

GERBEAUX Patrick

GEROLAMI/SANTANDREA René

GILBERT/ALESSI Marie-Christine

GIORGI Roch

GIOVANNI Antoine

GIRARD Nadine

GIRAUD/CHABROL Brigitte

GONCALVES Anthony
GRANEL/REY Brigitte

GRILLO Jean-Marie

GRIMAUD Jean-Charles

GROB Jean-Jacques

GUEDJ Eric

GUIEU Régis

GUIS Sandrine

GUYE Maxime

GUYOT Laurent

GUYS Jean-Michel

HABIB Gilbert

HARDWIGSEN Jean

HARLE Jean-Robert

HEIM Marc

HOFFART Louis

HOUVENAEGHEL Gilles

JACQUIER Alexis

JAMMES Yves Surnombre

JOLIVET/BADIER Monique

JOUVE Jean-Luc

KAPLANSKI Gilles

KARSENTY Gilles

KERBAUL François

KREITMANN Bernard

LAFFORGUE Pierre

LANCON Christophe

LA SCOLA Bernard

LAUGIER René

LAUNAY Franck

LAVIEILLE Jean-Pierre

LE CORROLLER Thomas

LE TREUT Yves-Patrice

LECHEVALLIER Eric

LEGRE Régis

LEONE Marc

LEONETTI Georges

LEPIDI Hubert

LEVY Nicolas

MACE LoÏC

MAGNAN Pierre-Edouard

MARANINCHI Dominique

MARTIN Claude

MEGE Jean-Louis

MERROT Thierry

METELLUS Philippe

MEYER/DUTOUR Anne

MICHEL Gérard

MICHELET Pierre

MONCLA Anne

MORANGE Pierre-Emmanuel

MOULIN GuY

MOUTARDIER Vincent

MUNDLER Olivier

NAUDIN Jean

NAZARIAN Serge Surnombre

NICOLLAS Richard

NICCOLI/SIRE Patricia

NICOLAS DE LAMBALLERIE Xavier

OLIVE Daniel

OREHEK Jean

OUAFIK L'Houcine

MAJ 01.11.2014 


\title{
PROFESSEURS DES UNIVERSITES-PRATICIENS HOSPITALIERS
}

CHARREL Rémi

PANUEL Michel PAPAZIAN Laurent PAROLA Philippe PARRATTE Sébastien PAUT Olivier PELISSIER-ALICOT Anne-Laure PELLETIER Jean PETIT Philippe PHAM Thao PIARROUX Renaud PIERCECCHI/MARTI Marie-Dominique PIQUET Philippe PIRRO Nicolas POINSO François POITOUT Dominique Surnombre POUGET Jean Surnombre RACCAH Denis RAOULT Didier REGIS Jean REYNAUD/GAUBERT Martine REYNAUD Rachel RICHARD/LALLEMAND Marie-Aleth
GORINCOUR Guillaume

PAGANELLI Franck

RIDINGS Bernard ROCHE Pierre-Hugues

ROCH Antoine ROCHWERGER Richard ROSSI Dominique ROSSI Pascal ROUDIER Jean SALAS Sébastien SAMBUC Roland SARLES Jacques SARLES/PHILIP Nicole SASTRE Bernard Surnombre SCAVARDA Didier SCHLEINITZ Nicolas SEBAG Frédéric SEITZ Jean-François SERMENT Gérard Surnombre SERRATRICE Jacques SIELEZNEFF Igor SIMON Nicolas STEIN Andréas TARANGER Colette
THIRION Xavier THOMAS Pascal THOMASSIN Jean-Marc Surnombre THUNY Franck TRIGLIA Jean-Michel TROPIANO Patrick TSIMARATOS Michel VACHER-COPONAT Henri VALERO René VEY Norbert VIALETTES Bernard Surnombre VIDAL Vincent VIENS Patrice VILLANI Patrick VITON Jean-Michel VITTON Véronique VIEHWEGER Heide Elke VIVIER Eric XERRI LuC

\section{PROFESSEUR DES UNIVERSITES}

\author{
ADALIAN Pascal \\ AGHABABIAN Valérie \\ BELIN Pascal \\ CHABANNON Christian \\ CHABRIERE Eric \\ FERON François \\ LE COZ Pierre \\ LEVASSEUR Anthony \\ RANJEVA Jean-Philippe \\ SOBOL Hagay
}

PROFESSEUR CERTIFIE

BRANDENBURGER Chantal

PRAG

TANTI-HARDOUIN Nicolas

PROFESSEUR ASSOCIE DE MEDECINE GENERALE A MI-TEMPS

DUMOND-HUSSON Monique FILIPPI Simon

PROFESSEUR ASSOCIE A TEMPS PARTIEL 


\section{MAITRES DE CONFERENCES DES UNIVERSITES-PRATICIENS HOSPITALIERS}

ACHARD Vincent ANDRE Nicolas

ATLAN Catherine BACCINI Véronique

BALIQUE Hubert BARTHELEMY Pierre

BARTOLI Christophe

BEGE Thierry

BELIARD Sophie

BERBIS Julie

BERGE-LEFRANC Jean-Louis

BLONDEL Benjamin

BONELLO Laurent

BOUCRAUT Joseph

BOULAMERY/VELLY Audrey

BOULLU/CIOCCA Sandrine

BREGEON Fabienne

BUFFAT Christophe

CALAS/AILLAUD Marie-Françoise

CAMILLERI Serge

CARRON Romain

CASTINETTI Frédéric

CHAUDET Hervé

CHICHEPORTICHE Colette

COURBIERE Blandine

COZE Carole

DADOUN Frédéric (disponibilité)

DAHAN ALCARAZ Laétitia

DALES Jean-Philippe

DEGEORGES/VITTE Joëlle

DEL VOLGO/GORI Marie-José

DELLIAUX Stéphane

DESPLAT/JEGO Sophie

DEVEZE Arnaud
DUFOUR Jean-Charles

FABRE Alexandre

FARAUT Françoise

FAUGERE Bernard

FOUILLOUX Virginie

FRERE Corinne

GABORIT Bénédicte

GASTALDI Marguerite

GAUDART Jean

GAUDY/MARQUESTE Caroline

GAVARET Martine

GELSI/BOYER Véronique

GIUSIANO COURCAMBECK Sophie

GOURIET Frédérique

GREILLIER Laurent

GRISOLI Dominique

GUIDON Catherine

HAUTIER/KRAHN Aurélie

HRAIECH Sami

JOURDE CHICHE Noémie

KRAHN Martin

LABIT/BOUVIER Corinne

LAFAGE/POCHITALOFF-HUVALE Marina

LAGIER Aude

LAGIER Jean-Christophe

LAGOUANELLE/SIMEONI Marie-Claude

LE HUCHER-MICHEL Marie-Pascale

LEJEUNE Pierre-Jean

LEVY/MOZZICONACCI Annie

LOOSVELD Marie

MANCINI Julien

MARGOTAT Alain

MARY Charles

MATONTI Frédéric
METZLER/GUILLEMAIN Catherine

MICALLEF/ROLL Joëlle

MICHEL Fabrice

MOTTOLA GHIGO Giovanna

NGUYEN PHONG Karine

NINOVE Laetitia

OUAISSI Medhi

PAULMYER/LACROIX Odile

PERRIN Jeanne

RANQUE Stéphane

REY Marc

ROBAGLIA/SCHLUPP Andrée

ROBERT Philippe

ROLL Patrice

SARI/MINODIER Irène

SARLON BARTOLI Gabrielle

SAVEANU Alexandru

SECQ Véronique

SOULA Gérard

TAIEB David

TOGA Caroline

TOGA Isabelle

TREBUCHON/DA FONSECA Agnès

TROUSSE Delphine

VALLI Marc

VELLY Lionel

VELY Frédéric

VION-DURY Jean

ZATTARA/CANNONI Hélène

\section{MAITRES DE CONFERENCES DES UNIVERSITES}

(mono-appartenants)

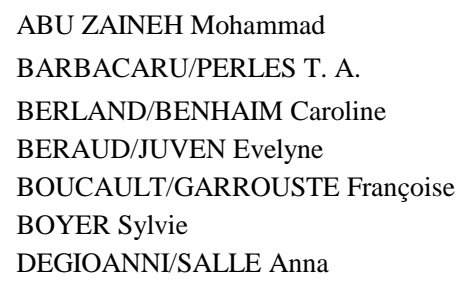

RUEL Jérôme STEINBERG Jean-Guillaume

THOLLON Lionel

THIRION Sylvie

MARANINCHI Marie

MERHEJ/CHAUVEAU Vicky

MINVIELLE/DEVICTOR Bénédicte

POGGI Marjorie

MAITRES DE CONFERENCES ASSOCIES DE MEDECINE GENERALE à MI-TEMPS

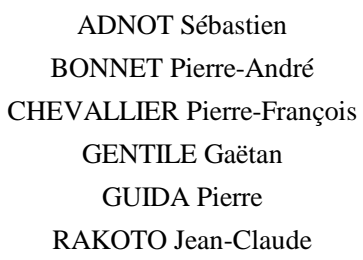


PROFESSEURS DES UNIVERSITES et MAITRES DE CONFERENCES DES UNIVERSITES - PRATICIENS HOSPITALIERS PROFESSEURS ASSOCIES, MAITRES DE CONFERENCES DES UNIVERSITES mono-appartenants

\section{ANATOMIE 4201}

ANTHROPOLOGIE 20

BRUNET Christian (PU-PH)

CHAMPSAUR Pierre (PU-PH)

LE CORROLLER Thomas (PU-PH)

NAZARIAN Serge (PU-PH) Surnombre

PIRRO Nicolas (PU-PH)

LAGIER Aude (MCU-PH)

THOLLON Lionel (MCF) (60ème section Service Pr BRUNET C.)
ADALIAN Pascal (PR)

DEGIOANNI/SALLE Anna (MCF)

BACTERIOLOGIE-VIROLOGIE ; HYGIENE HOSPITALIERE 4501

CHARREL Rémi (PU PH)

DE MICCO Philippe (PU-PH) Surnombre

DRANCOURT Michel (PU-PH)

FENOLLAR Florence (PU-PH)

FOURNIER Pierre-Edouard (PU-PH)

NICOLAS DE LAMBALLERIE Xavier (PU-PH)

LA SCOLA Bernard (PU-PH)

RAOULT Didier (PU-PH)

GOURIET Frédérique (MCU-PH)

NINOVE Laetitia (MCU-PH)

CHABRIERE Eric (PR) (64ème section)

LEVASSEUR Anthony (PR) (64ème section)

DESNUES Benoit (MCF) (65ème section)

MERHEJ/CHAUVEAU Vicky (MCF) (87ème section)

BIOCHIMIE ET BIOLOGIE MOLECULAIRE 4401

BARLIER/SETTI Anne (PU-PH)

ANESTHESIOLOGIE ET REANIMATION CHIRURGICALE ; MEDECINE URGENCE 4801

ENJALBERT Alain (PU-PH)

GABERT Jean (PU-PH)

GUIEU Régis (PU-PH)

OUAFIK L'Houcine (PU-PH)

\section{ALBANESE Jacques (PU-PH)}

AUFFRAY Jean-Pierre (PU-PH) Surnombre

BRUDER Nicolas (PU-PH)

KERBAUL François (PU-PH)

LEONE Marc (PU-PH)

MARTIN Claude (PU-PH)

MICHELET Pierre (PU-PH)

PAUT Olivier (PU-PH)

BUFFAT Christophe (MCU-PH)

MARGOTAT Alain (MCU-PH)

MOTTOLA GHIGO Giovanna (MCU-PH)

SAVEANU Alexandru (MCU-PH)

\section{GUIDON Catherine (MCU-PH)}

MICHEL Fabrice (MCU-PH)

VELLY Lionel (MCU-PH)

\section{BIOLOGIE CELLULAIRE 4403}

\section{ANGLAIS 11}

BRANDENBURGER Chantal (PRCE)

BURKHART Gary (PAST)
AUTILLO/TOUATI Amapola (PU-PH)

CAU Pierre (PU-PH) Surnombre

FONTES Michel (PU-PH)

GASTALDI Marguerite (MCU-PH)

LEVY/MOZZICONNACCI Annie (MCU-PH)

ROBAGLIA/SCHLUPP Andrée (MCU-PH)

ROLL Patrice (MCU-PH)

DUBOIS Christophe (MCF) (65ème section) 
GUEDJ Eric (PU-PH)

GUYE Maxime (PU-PH)

MUNDLER Olivier (PU-PH)

BELIN Pascal (PR) (69ème section)

RANJEVA Jean-Philippe (PR) (69ème section)

CAMMILLERI Serge (MCU-PH)

TAIEB David (MCU-PH)

VION-DURY Jean (MCU-PH)

BARBACARU/PERLES Téodora Adriana (MCF) (69ème section)

AVIERINOS Jean-François (PU-PH)

BONNET Jean-Louis (PU-PH)

CUISSET Thomas (PU-PH)

DEHARO Jean-Claude (PU-PH)

FRAISSE Alain (PU-PH)

HABIB Gilbert (PU-PH)

PAGANELLI Franck (PU-PH)

THUNY Franck (PU-PH)

BONELLO Laurent (MCU PH)

\section{CHIRURGIE DIGESTIVE 5202}

BERDAH Stéphane (PU-PH)

HARDWIGSEN Jean (PU-PH)

LE TREUT Yves-Patrice (PU-PH)

SASTRE Bernard (PU-PH) Surnombre

SIELEZNEFF Igor (PU-PH)

ET TECHNOLOGIES DE COMMUNICATION 4604

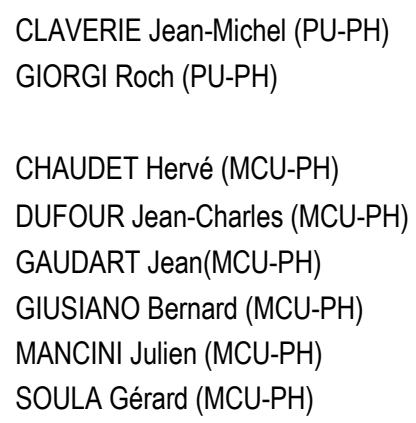

CLAVERIE Jean-Michel (PU-PH)

GIORGI Roch (PU-PH)

CHAUDET Hervé (MCU-PH)

DUFOUR Jean-Charles (MCU-PH)

GAUDART Jean(MCU-PH)

GIUSIANO Bernard (MCU-PH)

MANCINI Julien (MCU-PH)

SOULA Gérard (MCU-PH)

ABU ZAINEH Mohammad (MCF) (5ème section)

BOYER Sylvie (MCF) (5ème section)
OUAISSI Medhi (MCU-PH)

\section{CHIRURGIE GENERALE 5302}

DELPERO Jean-Robert (PU-PH)

MOUTARDIER Vincent (PU-PH)

SEBAG Frédéric (PU-PH)

BEGE Thierry (MCU-PH)

\section{CHIRURGIE ORTHOPEDIQUE ET TRAUMATOLOGIQUE 5002}

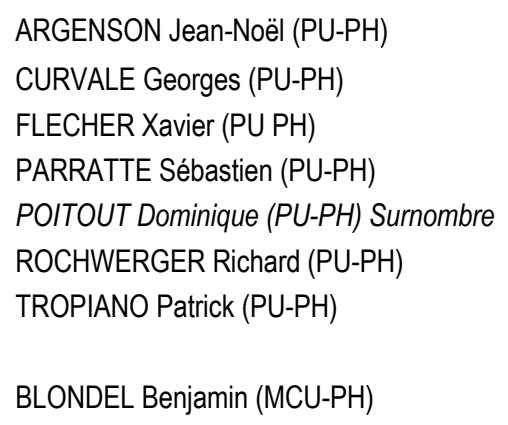

\section{CHIRURGIE INFANTILE 5402}

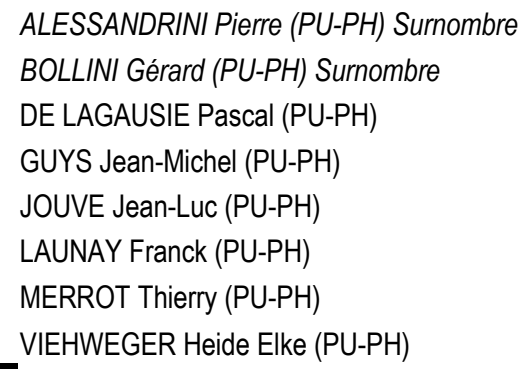

CHIRURGIE MAXILLO-FACIALE ET STOMATOLOGIE 5503

BLANC Jean-Louis (PU-PH) Surnombre

CHOSSEGROS Cyrille (PU-PH)

GUYOT Laurent (PU-PH) 
\begin{tabular}{|c|}
\hline CHIRURGIE THORACIQUE ET CARDIOVASCULAIRE 5103 \\
\hline
\end{tabular}

COLLART Frédéric (PU-PH)

D'JOURNO Xavier (PU-PH)

DODDOLI Christophe (PU-PH)

GARIBOLDI Vlad (PU-PH)

KREITMANN Bernard (PU-PH)

MACE Loïc (PU-PH)

THOMAS Pascal (PU-PH)

FOUILLOUX Virginie (MCU-PH)

GRISOLI Dominique (MCU-PH)

TROUSSE Delphine (MCU-PH)
CHIRURGIE PLASTIQUE,

RECONSTRUCTRICE ET ESTHETIQUE ; BRÛLOLOGIE 5004

BARDOT Jacques (PU-PH)

CASANOVA Dominique (PU-PH)

LEGRE Régis (PU-PH)

HAUTIER/KRAHN Aurélie (MCU-PH)

\begin{tabular}{l}
\hline \multicolumn{1}{|c|}{ CHIRURGIE VASCULAIRE ; MEDECINE VASCULAIRE 5104} \\
ALIMI Yves (PU-PH) \\
AMABILE Philippe (PU-PH) \\
BARTOLI Michel (PU-PH) \\
MAGNAN Pierre-Edouard (PU-PH) \\
PIQUET Philippe (PU-PH) \\
SARLON BARTOLI Gabrielle (MCU PH) \\
CYTOLOGIE ET HISTOLOGIE 4202
\end{tabular}

GRILLO Jean-Marie (PU-PH)

ACHARD Vincent (MCU-PH)

CHICHEPORTICHE Colette (MCU-PH)

LEPIDI Hubert (MCU-PH)

PAULMYER/LACROIX Odile (MCU-PH)
GASTROENTEROLOGIE ; HEPATOLOGIE ; ADDICTOLOGIE 5201

BARTHET Marc (PU-PH)

BERNARD Jean-Paul (PU-PH)

BOTTA/FRIDLUND Danielle (PU-PH)

GEROLAMI-SANTANDREA René (PU-PH)

GRIMAUD Jean-Charles (PU-PH)

LAUGIER René (PU-PH)

SEITZ Jean-François (PU-PH)

VITTON Véronique (PU-PH)

DAHAN ALCARAZ Laetitia (MCU PH)

GENETIQUE 4704

BEROUD Christophe (PU-PH)

LEVY Nicolas (PU-PH)

MONCLA Anne (PU-PH)

SARLES/PHILIP Nicole (PU-PH)

GROB Jean-Jacques (PU-PH)

RICHARD/LALLEMAND Marie-Aleth (PU-PH)

KRAHN Martin (MCU-PH)

NGYUEN Karine (MCU-PH)

TOGA Caroline (MCU-PH)

ZATTARA/CANNONI Hélène (MCU-PH)

\section{GYNECOLOGIE MEDICALE 5404}

BRUE Thierry (PU-PH)

CONTE-DEVOLX Bernard (PU-PH) surnombre

NICCOLI/SIRE Patricia (PU-PH)

CASTINETTI Frédéric (MCU-PH)

GYNECOLOGIE-OBSTETRIQUE ; GYNECOLOGIE MEDICALE 5403

EPIDEMIOLOGIE, ECONOMIE DE LA SANTE ET PREVENTION 4601

AGOSTINI Aubert (PU-PH)

AUQUIER Pascal (PU-PH)

CHABOT Jean-Michel (PU-PH)

GENTILE Stéphanie (PU-PH)

BOUBLI Léon (PU-PH)

BRETELLE Florence (PU-PH)

SAMBUC Roland (PU-PH)

CRAVELLO Ludovic (PU-PH)

THIRION Xavier (PU-PH)

D'ERCOLE Claude (PU-PH)

BALIQUE Hubert (MCU-PH)

COURBIERE Blandine (MCU-PH)

BERBIS Julie (MCU-PH)

LAGOUANELLE/SIMEONI Marie-Claude (MCU-PH)

MINVIELLE/DEVICTOR Bénédicte (MCF)(06ème section) 
BONGRAND Pierre (PU-PH)

KAPLANSKI Gilles (PU-PH)

MEGE Jean-Louis (PU-PH)

OLIVE Daniel (PU-PH)

VIVIER Eric (PU-PH)

FERON François (PR) (69ème section)

BOUCRAUT Joseph (MCU-PH)

DEGEORGES/VITTE Joëlle (MCU-PH)

DESPLAT/JEGO Sophie (MCU-PH)

ROBERT Philippe (MCU-PH)

VELY Frédéric (MCU-PH)

BERAUD/JUVEN Evelyne (MCF) 65ème section)

BOUCAULT/GARROUSTE Françoise (MCF) 65ème section)
BLAISE Didier (PU-PH)

COSTELLO Régis (PU-PH)

CHIARONI Jacques (PU-PH)

GILBERT/ALESSI Marie-Christine (PU-PH)

MORANGE Pierre-Emmanuel (PU-PH)

VEY Norbert (PU-PH)

BACCINI Véronique (MCU-PH)

CALAS/AILLAUD Marie-Françoise (MCU-PH)

FRERE Corinne (MCU-PH)

GELSI/BOYER Véronique (MCU-PH)

LAFAGE/POCHITALOFF-HUVALE Marina (MCU-PH)

LOOSVELD Marie (MCU-PH)

POGGI Marjorie (MCF) (64ème section)

\section{MEDECINE LEGALE ET DROIT DE LA SANTE 4603}

LEONETTI Georges (PU-PH)

PELISSIER/ALICOT Anne-Laure (PU-PH)

MALADIES INFECTIEUSES ; MALADIES TROPICALES 4503

BARTOLI Christophe (MCU-PH)

BERLAND/BENHAIM Caroline (MCF) (1ère section)

PAROLA Philippe (PU-PH)

STEIN Andréas (PU-PH)

MEDECINE PHYSIQUE ET DE READAPTATION 4905

LAGIER Jean-Christophe (MCU-PH)

MEDECINE INTERNE ; GERIATRIE ET BIOLOGIE DU

BENSOUSSAN Laurent (PU-PH)

DELARQUE Alain (PU-PH)

VIEILLISSEMENT ; MEDECINE GENERALE ; ADDICTOLOGIE 5301

VITON Jean-Michel (PU-PH)

BONIN/GUILLAUME Sylvie (PU-PH)

DISDIER Patrick (PU-PH)

DURAND Jean-Marc (PU-PH)

FRANCES Yves (PU-PH)

MEDECINE ET SANTE AU TRAVAIL 4602

GRANEL/REY Brigitte (PU-PH)

HARLE Jean-Robert (PU-PH)

ROSSI Pascal (PU-PH)

SCHLEINITZ Nicolas (PU-PH

SERRATRICE Jacques (PU-PH)

LEHUCHER/MICHEL Marie-Pascale (MCU-PH)

SARI/MINODIER Irène (MCU-PH)

FILIPPI Simon (PR associé Méd. Gén. à mi-temps)

ADNOT Sébastien (MCF associé Méd. Gén. à mi-temps)

BONNET Pierre-André (MCF associé Méd. Gén à mi-temps)

CHEVALLIER Pierre-François (MCF associé Méd. Gén. à mi-temps)

GENTILE Gaëtan (MCF associé Méd. Gén. à mi-temps)

GUIDA Pierre (MCF associé Méd. Gén. à mi-temps)

BOTTA Alain (PU-PH) Surnombre

BERGE-LEFRANC Jean-Louis (MCU-PH)

BERLAND Yvon (PU-PH)

BRUNET Philippe (PU-PH)

BURTEY Stépahne (PU-PH)

DUSSOL Bertrand (PU-PH)

TSIMARATOS Michel (PU-PH)

VACHER-COPONAT Henri (PU-PH)

JOURDE CHICHE Noémie (MCU PH) 


\begin{tabular}{ll}
\hline \multicolumn{1}{|c|}{ NUTRITION 4404 } & NEUROC \\
DARMON Patrice (PU-PH) & \\
RACCAH Denis (PU-PH) & DUFOUR Henry (PU-PH) \\
VALERO René (PU-PH) & FUENTES Stéphane (PU-PH) \\
VIALETTES Bernard (PU-PH) Surnombre & METELLUS Philippe (PU-PH) \\
ATLAN Catherine (MCU-PH) & REGIS Jean (PU-PH) \\
BELIARD Sophie (MCU-PH) & ROCHE Pierre-Hugues (PU-PH) \\
MARANINCHI Marie (MCF) (66ème section) & SCAVARDA Didier (PU-PH) \\
\end{tabular}

\section{ONCOLOGIE 65 (BIOLOGIE CELLULAIRE)}

CHABANNON Christian (PR) (66ème section)

ATTARIAN Sharham (PU PH)

SOBOL Hagay (PR) (65ème section)

AUDOIN Bertrand (PU-PH)

AZULAY Jean-Philippe (PU-PH)

CECCALDI Mathieu (PU-PH)

FELICIAN Olivier (PU-PH)

NICOLI François (PU-PH)

PELLETIER Jean (PU-PH)

POUGET Jean (PU-PH) Surnombre
DENIS Danièle (PU-PH)

HOFFART Louis (PU-PH)

RIDINGS Bernard (PU-PH)

MATONTI Frédéric (MCU-PH)
PEDOPSYCHIATRIE; ADDICTOLOGIE 4904

DA FONSECA David (PU-PH)

POINSO François (PU-PH)

\section{OTO-RHINO-LARYNGOLOGIE 5501}

DESSI Patrick (PU-PH)

GIOVANNI Antoine (PU-PH)

LAVIEILLE Jean-Pierre (PU-PH)

NICOLLAS Richard (PU-PH)

THOMASSIN Jean-Marc (PU-PH) Surnombre

TRIGLIA Jean-Michel (PU-PH)

DEVEZE Arnaud (MCU-PH)

REVIS Joana (MAST) (Orthophonie) (7ème Section)
PHARMACOLOGIE FONDAMENTALE -

PHARMACOLOGIE CLINIQUE; ADDICTOLOGIE 4803

\author{
BLIN Olivier (PU-PH) \\ FAUGERE Gérard (PU-PH) \\ SIMON Nicolas (PU-PH)
}

BOULAMERY/VELLY Audrey (MCU-PH)

MICALLEF/ROLL Joëlle (MCU-PH)

VALLI Marc (MCU-PH)

\section{PARASITOLOGIE ET MYCOLOGIE 4502}

\section{DESSEIN Alain (PU-PH)}

DUMON Henri (PU-PH) en surnombre

PIARROUX Renaud (PU-PH)

FARAUT Françoise (MCU-PH)

FAUGERE Bernard (MCU-PH)

MARY Charles (MCU-PH)

RANQUE Stéphane (MCU-PH)

TOGA Isabelle (MCU-PH)

\section{PHILOSPHIE 17}

LE COZ Pierre (PR) (17ème section)

ALTAVILLA Annagrazia (PR Associé à mi-temps) 


\section{Remerciements}

Au Docteur Ronzier, sans qui je ne serais jamais devenue médecin. Admirative de votre gentillesse et votre professionnalisme, j'ai débuté mes études de médecine en rêvant de devenir pédiatre comme vous. Finalement mon choix se portera sur la gastro-entérologie en espérant avoir une aussi longue carrière que la vôtre et une patientèle aussi fidèle. Et j'espère moi aussi un jour peut-être inspirer quelqu'un à faire ce beau métier.

A Monsieur le Professeur Grimaud pour me faire l'honneur de présider mon jury de thèse et à Monsieur le Professeur Barthet pour ses relectures attentives (jusqu'à la moindre virgule) de ma thèse. Merci pour ces deux semestres d'internat dans votre service. Grâce à vous j'ai appris (la physiologie digestive !!) et progressé pour passer de jeune interne à future assistante et j'espère bien profiter de ces deux années à venir à vos côtés pour me perfectionner encore. Veuillez trouver ici le témoignage de ma gratitude et de mon profond respect.

A Monsieur le Docteur Gasmi, pour avoir accepté d'être mon directeur de thèse. Merci de m'avoir conforté dans mon choix d'oncologie digestive et de m'avoir prouvé qu'on peut y allier un excellent travail d'endoscopiste. Merci pour ta confiance et tes conseils.

A Messieurs les Professeurs D'journo et Leone qui ont accepté de faire partie de mon jury et de juger mon travail. Veuillez trouver ici l'expression de ma sincère reconnaissance. 
Au Professeur Vitton et au Dr Desjeux du service de gastroentérologie de l'hôpital Nord. Merci pour tout ce que vous m'avez transmis pendant ces deux semestres ô combien formateurs.

Aux Professeurs Seitz, Laugier, Bernard, Botta, et Gérolami. Les différents stages effectués dans vos services m'ont permis de grandir en tant que médecin.

A Monsieur le Pr Raoul, aventurier et puit sans fond de connaissances.

Aux Docteurs Dahan, Grandval, Heyriès, Borentin, Portal, Gilabert, Ries, Oziel-Taïeb, Dermeche, Bernardini, Ah-Soune, Chinoune, Le Goffic, Mercky, Aider, Mallaret, Cougard, Giovannini, Bories, Pesenti, Caillol et Tournut pour votre présence, votre aide, votre bonne humeur durant ces 4 années d'internat.

A Gaëlle, Clio, Sophie, Cécile et Saida pour m'avoir montré qu'on peut jongler entre son job de gastro-entérologue, de maman, d'aventurière, d'amie et garder les pieds sur terre.

A Jean-Philippe et Jean-Michel, mes "supers-assistants". Merci pour tout ce que vous m'avez appris, votre "zénitude ", votre assurance. Jean-mi merci pour ton aide précieuse pour ma thèse.

A la team du $9^{\text {ème }}$ A : Anne-Marie, Hélénus, Laure, Aurélie, Cindy, Miled, Mariama, Gersende, Julia, Margaux, et Nabil. Deux semestres au top avec vous et on se retrouve bientôt.

A mes co-internes, ceux que je vois encore, ceux qui ont disparu, à nos peurs des premiers jours, à nos délires, aux moments plus durs: Philippe, Astrid, Guillaume, Edouardo, Sylvain, Alban, Xavier, Ryma, Maxime, Sébastien, Hortense, Nastassia, Thomas et Guigui (alias Tic et Tac), Gregot le Payot, Marthus, Marine, Arnaud, Isabelle. 
A Lola, ton excentricité et ta joie de vivre ont égayé les moments passés ensemble depuis le premier jour de la P1 jusqu'à maintenant. C'est d'ailleurs un SCANDALE que tu sois si loin de moi !!!

A Jérémy, aux soirées passées ensemble, à nos vagabondages au bout du monde, à nos différences qui nous rapprochent, à nos futurs voyages et autres aventures qui nous attendent (mais par pitié pas comme la nuit à Lisbonne !!). "Because I knew you... »

A Lucie, tu as eu l'idée, j'avais les DVD, l'AMPciné est né puis s'est effondré, mais on s'en fout car ça a continué : les vacances à l'lle de Ré, la gâche et le caramel au beurre salé, les soirées déguisées, tes anniversaires décalés (bientôt 25 ans ??!!).

A Amélie, parce qu'on passe plus de temps à se rater qu'à s'avoir au téléphone mais quand l'une ou l'autre décroche c'est comme si nous nous étions quittées la veille, pour rigoler et se confier, râler, se disputer.

A Vincent, merci de me contrebalancer avec ta décontraction permanente, merci d'être là pour Toudou, merci d'avoir été habiter au ski!

Aux copains de médecine, la team formée autour du baby-foot : Matthieu, GDV, François, Anouck, Julien, Marion, Elodie. Toujours présents dans les bons et les mauvais moments.

A mes amis fidèles du collège, Marc et Julien, toujours disponibles lors de mes retours sur Paris pour ressasser les vieux souvenirs et rattraper les nouvelles plus fraiches.

A Axel, il sait pourquoi. 
A mes cousines Livia, Marc-Antonia et Ghjulia-Maria. Je me suis rapprochée mais nous sommes encore trop loin et le temps passé ensemble est toujours trop court.

A mon oncle Marc, merci de m'avoir conseillé « d'aller prendre l'air » à Marseille. A ma tante Marie-Jeanne merci de me donner l'envie de ne jamais quitter la Corse après les repas en famille à Alata.

A ma famille marseillaise, Marie, Pierre, Odette, les streums, ça a été moins dur de quitter Paris grâce à vous.

A Jocelyne, merci de ne pas trop me détester d'avoir emmené Julien dans mon périple marseillais. A Christian, merci de ne pas trop m'en vouloir lorsque Nadal remportera encore RollandGarros cette année.

A Jeannot Tagnati, l'un de mes plus fidèles supporters durant ces longues années de médecine et à sa femme Dominique pour sa douceur et sa gentillesse. A Indji pour ses conseils.

Aux marseillais : Sandra et Yoann pour votre amitié depuis mon arrivée dans la cité phocéenne, nos dîners, les soirées foot et les soirées filles. Floriane et Jean, à votre bonne humeur, aux futures soirées chez vous, à la mascotte Clarisse.

A Soraya et Chiara, merci d'être venues visiter notre belle ville de Marseille. Merci pour les fous rires, pour nos discussions, pour mes futures vacances chez vous.

A Philippe et Muriel pour m'avoir reçu lorsque j'ai émigré à Toulon pendant six rudes mois d'hiver.

A Mr Tissot mon professeur de mathématiques du lycée qui a fini de me convaincre que d'être différente c'est ce qui compte. Vivre ses rêves et ne pas rêver sa vie. 
A mes parents qui ont toujours été à mes côtés durant toutes ces années, sans qui je ne serais pas où je suis ni qui je suis aujourd'hui. Merci à ma maman de s'être battue pour rester à nos côtés. Merci à mon papa de m'avoir transmis son amour des films. Merci pour l'éducation que vous nous avez donnée et les valeurs que vous nous avez transmises. Je suis heureuse de partager cette journée avec vous. Merci de tout cœur pour tout. Je vous aime.

A mon frère Antoine, qui malgré les $800 \mathrm{k} m$ qui nous séparent est resté mon confident. A notre enfance, à nos bagarres, à tes lunettes cassées, à nos délires. Arrête de me parler de tes " jurisprudences » et j'arrêterai de te parler de mes " coloscopies".

A Edouard et Marie, qui grâce à Antoine font partie de ma famille maintenant.

Aux câlins de mamie Françoise, au goéland de mamie Nanou, aux histoires de papy Nini et aux chansons de Babo Simon. Aucun de vous n'est là en ce jour particulier mais je sais que de là où vous êtes, vous devez être fiers.

A Misgio, Pipo, Grosse Rougne et surtout ma Chibi Marou. 
A Julien, merci pour ta patience et ton soutien durant ces sept années. Tu es ma bulle d'air et mon meilleur ami. Je t'aime et j'ai hâte de passer le reste de ma vie avec toi. Vivement le 26 Septembre. 


\section{SOMMAIRE}

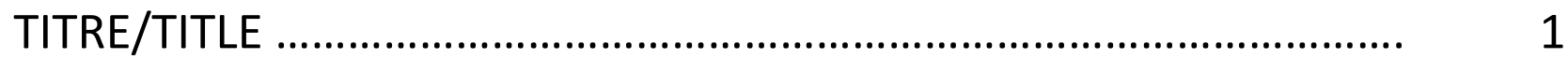

RESUME ....................................................................... 2

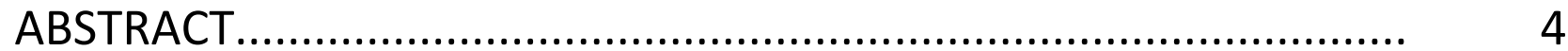

INTRODUCTION ..................................................................... 6

MATERIELS ET METHODES....................................................... 7

Patients et diagnostic de fistule ..............................................

Objectifs et définitions ........................................................ 8

Procédure endoscopique et stratégie ...................................... 8

Analyse statistique ..................................................... 9

RESULTATS ...................................................................... 10

Caractéristiques des patients ............................................. 10

Diagnostic et caractéristiques des fistules ................................. 11

Objectif principal ................................................................ 11

Objectifs secondaires ....................................................... 12

Analyse univariée en sous-groupe pour l'efficacité du traitement ...... 13

Analyse multivariée pour l'efficacité du traitement ...................... 15

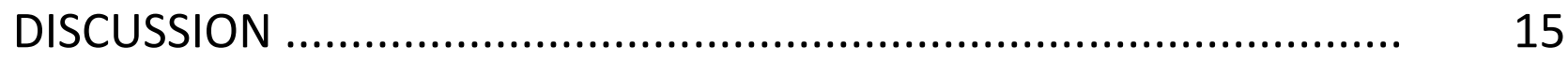

CONCLUSION...................................................................... 19

REFERENCES ................................................................. 20

TABLEAUX ......................................................................... 25 


\title{
Efficacité du traitement endoscopique dans la prise en charge des fistules digestives anastomotiques après chirurgie pour cancer de l'œsophage
}

\author{
ENDOSCOPIC MANAGEMENT OF POST-OPERATIVE LEAKAGES AND FISTULAS AFTER ESOPHAGEAL \\ ONCOLOGIC SURGERY: EVALUATION OF EFFICACY IN A LARGE RETROSPECTIVE STUDY
}

Auteurs:

C. Servajean ${ }^{1}$, J.-M. Gonzalez ${ }^{1}$, M. Gasmi ${ }^{1}$, B. X. D'Journo ${ }^{2}$, M. Leone ${ }^{3}$, J.-C. Grimaud ${ }^{1}$, M. Barthet ${ }^{1}$

${ }^{1}$ Gastroenterologie, AP-HM, Hôpital Nord, Marseille, France

${ }^{2}$ Chirurgie Thoracique, AP-HM, Hôpital Nord, Marseille, France

${ }^{3}$ Réanimation Médicale, AP-HM, Hôpital Nord, Marseille, France

\section{Correspondance :}

Cecilia Servajean Defranchi

63 rue Edmond Rostand, 13006 Marseille

Téléphone : 0672367687

Ceciliaservajeandefranchi@gmail.com

Mots-clés : Fistules anastomotiques, chirurgie œsophagienne, endoscopie interventionnelle.

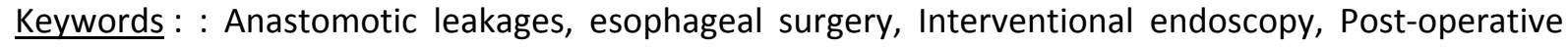
fistulas, Stenting. 


\section{RESUME}

Introduction La fistule anastomotique est l'une des principales complications de l'œsophagectomie pour cancer de l'œsophage ou de la jonction œso-cardiale. Elle serait responsable de $40 \%$ des décès en post-opératoire de cette chirurgie. Depuis 10 ans, la prise en charge endoscopique de ces fistules est devenue une alternative face à la reprise chirurgicale ou au traitement conservateur. L'objectif de cette étude est d'évaluer l'efficacité et les caractéristiques du traitement endoscopique dans cette indication.

Matériel et Méthodes Dans cette étude monocentrique rétrospective, 35 patients inclus consécutivement ont été traités endoscopiquement pour une fistule anastomotique après œsophagectomie pour cancer de l'œsophage ou de la jonction œso-cardiale entre juin 2010 et juin 2014. Toutes les procédures endoscopiques ont été réalisées dans l'unité d'endoscopie de l'hôpital Nord à Marseille, par des endoscopistes interventionnels, chez des patients intubés sous anesthésie générale, avec un endoscope à large canal opérateur (3,8mm, Pentax, Japon) sous contrôle scopique. Un contrôle endoscopique était systématiquement effectué 4 à 8 semaines après afin de s'assurer de l'efficacité ou de la nécessité d'un traitement additionnel. L'efficacité primaire et secondaire, le délai entre la chirurgie, le diagnostic et l'endoscopie, le nombre de reprises endoscopiques, le matériel utilisé (prothèse, clip, drain), les complications et le taux de mortalité ont été évalués et une analyse uni et multivariée a été réalisée afin de mettre en évidence des facteurs prédictifs de succès.

Résultats Parmi les 35 patients, il y avait 4 femmes et 31 hommes avec un âge moyen de 61,7 ans \pm 8,9 [43-85]. Les principales techniques chirurgicales employées ont été celles de Lewis-Santy pour $48,6 \%$ des cas et de Akiyama pour $45,7 \%$. $71,4 \%$ des patients avaient bénéficié d'une radiochimiothérapie néoadjuvante et $77,1 \%$ étaient hospitalisés en réanimation. Le délai médian entre la chirurgie et le premier traitement endoscopique était de 8,5 jours (IQR ; 6,00-18,25). 88,6\% des patients ont été traités par une prothèse métallique œsophagienne avec un taux final d'extirpabilité de $100 \%$ et un taux de migration de $18 \%$. Le nombre moyen d'endoscopies réalisées par patient a été de 2,6 \pm 1,57 [1-10] avec un nombre moyen de prothèses œsophagiennes de 1,6 \pm 1,35 [0-7] par patient. L'efficacité primaire du traitement endoscopique a été de $48,6 \%$ et l'efficacité finale de $68,6 \%$. Le taux de mortalité en cas de traitement endoscopique a été de $17 \%$, aucun décès n'étant lié au traitement endoscopique par lui-même. Le type de technique chirurgicale, la réalisation d'une radiochimiothérapie pré-opératoire et la taille de l'orifice fistuleux n'ont pas eu d'influence sur l'efficacité du traitement endoscopique que ce soit en analyse uni ou multivariée. 
Conclusion La prise en charge endoscopique des fistules anastomotiques post-œsophagectomie est efficace, en particulier avec les prothèses œsophagiennes métalliques et n'entraine aucune complication liée au traitement. Elle est de plus associée à un taux de mortalité de $17 \%$ inférieur à celui de la reprise chirurgicale ou du traitement conservateur (40 à 100\%) (1).

\section{Références}

1. Alanezi K, Urschel JD. Mortality secondary to esophageal anastomotic leak. Ann Thorac Cardiovasc Surg Off J Assoc Thorac Cardiovasc Surg Asia. avr 2004;10(2):71-5. 


\section{ABSTRACT}

Introduction Anastomotic leakages or fistulas are among the most common and severe complications of esophagectomy for esophageal cancer, with high grade of mortality. Recently the endoscopic management has taken a growing place in the treatment of digestive post-operative complications (1). The aim of this study was to evaluate the effectiveness and the characteristics of the endoscopic management in this indication.

Aims \& Methods This is a monocentric study on consecutive patients treated surgically in our institution between 2010 and 2014 for esophageal carcinomas. During this period, on 126 patients operated, 35 developed post-operative fistulas or leakages (27\%), endoscopically managed and included in this study. All the procedures were performed in our endoscopy unit, by interventional endoscopists, in intubated patients under general anesthesia, using a large operating channel gastroscope (3.8mm, Pentax, Japan) and fluoroscopy. The patients were systematically controlled endoscopically 6 weeks after the endoscopic treatment to check the efficacy out and to evaluate the need for an additional treatment. The primary or secondary efficacy, the time between surgery, diagnostic and endoscopy, the number of procedures, the material used (stents, clips, or drains), the complications, and the death rate were recorded, and uni- and multivariate analysis was carried out to determine predictive factors of success.

Results There were 4 women and 31 men, with a median age of 61.7 years \pm 8.9 [43-85]. The surgical techniques were in majority Lewis-Santi for $48.6 \%$ of cases, Akiyama for $45.7 \%$. $71.4 \%$ patients have undergone neo-adjuvant chemoradiation therapy and $77.1 \%$ were hospitalized in intensive care unit. The median delay between surgery and first endoscopy was 8.5 days [6.0018.25]. $88 \%$ of the patients were treated using metallic (double type) esophageal stents, with a removability rate of $100 \%$ and a migration rate of $18 \%$. The other ones were treated by Over-thescope clips, nasocystic drain or combined approach. The mean number of endoscopy per patient was $2.6 \pm 1.57$ [1-10], with a mean number of $1.6 \pm 1.35$ [0-7] stents placed. The primary efficacy of the endoscopic treatment was $48.6 \%$, the final efficacy was $68.6 \%$. The mortality rate in patients endoscopically managed was $17 \%$, none being related to procedures. No predictive risk factor of success or failure of the endoscopic treatment (CRT, type of surgery, fistula size, age...) could be identified in univariate as well as in multivariate analysis.

Conclusion The endoscopic management of leakages or fistulas after esophageal surgery is feasible, and lead to an overall effectiveness rate of $68.8 \%$. There were no significant complications related to 
the procedures, and the mortality rate was decreased to $17 \%$ compared to 40 to $100 \%$ rate in the literature (2). Self-expandable metallic stents are the most common used treatment with a removability rate of $100 \%$ and a migration rate of $18 \%$.

\section{References}

1. Gonzalez J-M, et al. Double-type metallic stents efficacy for the management of post-operative fistulas, leakages, and perforations of the upper gastrointestinal tract. Surg Endosc. 2014

2. Alanezi K, et al. Mortality secondary to esophageal anastomotic leak. Ann Thorac Cardiovasc Surg Off J Assoc Thorac Cardiovasc Surg Asia. 2004 


\section{INTRODUCTION}

Le cancer de l'œsophage est au $9^{\text {ème }}$ rang mondial en termes de fréquence et au $7^{\text {ème }}$ rang en termes de mortalité (1). C'est un cancer agressif dont la découverte est souvent tardive devant des symptômes de dysphagie signant une lésion déjà avancée. En effet, au diagnostic, seulement 20\% des patients ont une maladie localisée qui pourra être guérie. Actuellement le taux de survie à 5 ans tous stades confondus est de $17 \%(2)$.

Le traitement chirurgical par œsophagectomie reste le traitement de référence des cancers de l'œsophage localisés (stades I et II) même si le développement de la résection endoscopique des lésions superficielles par mucosectomie ou dissection sous-muqueuse a fait diminuer l'indication de la chirurgie pour les stades IA (3). Dans les stades localement avancés, la prise en charge optimale reste controversée (4). Cependant, l'œsophagectomie garde sa place après un traitement néoadjuvant ou en rattrapage en cas de persistance de cellules tumorales ou de récidive après une radiochimiothérapie exclusive (5). II existe alors plusieurs approches chirurgicales qui peuvent être divisées en deux catégories: la voie transthoracique (qui comprend les chirurgies de type LewisSanty et de type Akiyama) et la voie transhiatale. Ces deux approches ont montré le même taux de mortalité péri-opératoire et de survie à 5 ans (6-8). La chirurgie selon Lewis-Santy réalisée en deux temps associe une laparotomie et une thoracotomie droite avec une anastomose thoracique et ses indications sont les cancers du tiers moyen et du tiers inférieur de l'œsophage. La chirurgie selon Akiyama associe une troisième voie d'abord par cervicotomie gauche avec une anastomose cervicale. Elle est indiquée dans les cancers du tiers supérieur de l'œsophage (en majorité des carcinomes épidermoïdes) et les adénocarcinomes à cellules indépendantes peu importe leur localisation sur l'œsophage.

Or, l'une des principales complications de l'œsophagectomie est l'apparition d'une fistule anastomotique. Celle-ci survient après une chirurgie pour cancer de l'œsophage dans 5 à 20\% des cas selon les séries publiées $(9,10)$, et peut elle-même se compliquer d'une médiastinite, d'une infection pulmonaire voire d'un sepsis sévère. Elle serait responsable d'environ $40 \%$ des décès en postopératoire de cette chirurgie (9). Les deux principaux facteurs identifiés prédisposant à l'apparition d'une fistule anastomotique sont l'ischémie du conduit gastrique et un apport diminué en oxygène au niveau de la micro-circulation anastomotique d'où l'importance de la gestion péri-opératoire notamment anesthésique $(10,11)$.

La prise en charge de ces fistules reste mal codifiée. Jusqu'à il y a une dizaine d'années, les deux méthodes employées pour pallier à cette complication étaient la reprise chirurgicale et le traitement médical conservateur pouvant associer une antibiothérapie large spectre, une nutrition 
parentérale et un drainage péri-anastomotique. Cependant leurs taux de mortalité élevés, respectivement de $60-100 \%(9,10)$ et de $40 \%(12)$, ont poussé les équipes médico-chirurgicales à envisager d'autres options thérapeutiques. En effet, dans le même temps et depuis quelques années, la prise en charge endoscopique des fistules anastomotiques s'est particulièrement développée et a démontré son efficacité notamment dans le traitement des fistules compliquant la chirurgie bariatrique (13). On retrouve ainsi plusieurs articles soulignant l'efficacité des clips en particulier des clips larges $\operatorname{OVESCO}^{\odot}$ (Ovesco, Tubingen, Allemagne) dans la fermeture des fistules du tractus digestif supérieur $(13,14)$. En parallèle, l'usage des prothèses œsophagiennes couvertes s'est développé dans cette indication en découlant de la prise en charge des cancers fistulisés ou perforés inopérables (15) et a montré des résultats prometteurs (12,16-20) avec une efficacité variant de 60 (21) à 100\% (17). Cependant, aux vues du taux de migration élevé de ce type de stent, les prothèses œesophagiennes double-types sont devenues une alternative intéressante $(22,23)$.

Le but de cette étude était d'évaluer l'efficacité du traitement endoscopique des fistules anastomotiques après œsophagectomie pour des cancers de l'œsophage sur une série de 35 patients et d'essayer de mettre en évidence des facteurs influençant l'efficacité du traitement.

\section{MATERIELS ET METHODES}

Il s'agit d'une étude rétrospective mono-centrique menée dans les services de gastroentérologie, de chirurgie thoracique et d'endoscopie digestive de l'Hôpital Nord à Marseille. Tous les patients opérés entre juin 2010 et juin 2014 par l'équipe de chirurgie thoracique pour un cancer de l'œsophage ou de la jonction œso-cardiale ont été considérés. Les patients qui ont présenté dans les suites opératoires une fistule digestive haute et qui ont bénéficié d'une endoscopie thérapeutique ont été inclus dans l'étude et finalement analysés. L'objectif global de l'étude était d'évaluer l'efficacité du traitement endoscopique d'une fistule après œsophagectomie.

\section{Patients et diagnostic de fistule}

Pour chaque patient inclus étaient relevés l'âge, le sexe, l'existence d'un traitement néoadjuvant éventuel (chimiothérapie ou radio-chimiothérapie), le type histologique du cancer, le stade TN sur la pièce de résection ainsi que les marges de résection (RO-R1), et le type d'intervention chirurgicale réalisée (par double voie d'abord, dite de type Lewis-Santy, ou par triple voie d'abord, dite de type Akiyama). On notait également le délai entre la chirurgie et le premier traitement endoscopique (comprenant le délai entre la chirurgie et le diagnostic de fistule et le délai entre le 
diagnostic de fistule et la réalisation du premier traitement endoscopique, si le diagnostic était fait lors d'un examen radiologique), le lieu d'hospitalisation en réanimation ou en service au moment du traitement endoscopique, le délai de guérison à partir du diagnostic initial de fistule, le nombre de reprises endoscopiques total.

L'existence d'une fistule était suspectée devant l'apparition d'un sepsis sévère, d'une dégradation respiratoire et /ou d'une modification de l'aspect et du volume d'écoulement par les drains thoraciques. Le diagnostic de fistule était parfois évoqué par un examen d'imagerie précédant l'endoscopie (transit œso-gastro-duodénal ou scanner thoraco-abdominal sans et avec injection) et était définitivement confirmé lors de l'endoscopie elle-même.

\section{Objectifs et définitions}

L'objectif principal de cette étude était d'évaluer l'efficacité du traitement endoscopique des fistules ou lâchage anastomotiques après chirurgie œsophagienne. Les critères d'évaluation étaient l'efficacité primaire et secondaire de la prise en charge endoscopique. L'efficacité primaire était définie par la guérison d’une fistule/lâchage confirmée par un contrôle endoscopique avec opacification sans récidive secondaire après une seule session de traitement endoscopique. L'efficacité secondaire était définie par la guérison finale de cette fistule/lâchage après plus d'un traitement endoscopique. L'échec était défini par la nécessité d'une reprise chirurgicale ou le décès du patient.

Les objectifs secondaires étaient d'évaluer le délai de guérison à partir de la chirurgie initiale et du diagnostic de fistule, le taux de complication (migration, extirpabilité, hémorragie...) du traitement, le nombre moyen d'interventions endoscopiques permettant une efficacité secondaire, la mise en évidence de facteurs influençant cette efficacité en analyse uni et multivariée (type de chirurgie, réalisation d’un traitement néo-adjuvant, taille de la fistule).

\section{Procédure endoscopique et stratégie}

Les endoscopies œso-gastro-duodénales se déroulaient sous anesthésie générale, après intubation oro-trachéale et sous contrôle radioscopique. Les procédures ont été réalisées sur table de scopie par des endoscopistes interventionnels avec un endoscope à large canal opérateur $(3,8 \mathrm{~mm}$, Pentax, Japon) et un insufflateur à $\mathrm{CO} 2$ lorsque disponible. L'endoscopie comprenait 2 phases : diagnostique puis thérapeutique. La phase diagnostique consistait en la confirmation et la caractérisation de la fistule ou du lâchage anastomotique soit directement par la vision endoscopique soit par un contrôle radioscopique après opacification par un produit de contraste iodé. La taille de l'orifice était alors évaluée ainsi que sa hauteur par rapport aux arcades dentaires, 
l'aspect nécrotique, inflammatoire ou fibreux de ses berges et la présence de pus et d'une collection associée non drainée. Ensuite, la prise en charge thérapeutique était réalisée, parfois dans le même temps opératoire, après discussion de la stratégie thérapeutique par la mise en place d'une prothèse métallique couverte, d'un clip ou encore d'un traitement combiné afin de recouvrir ou de fermer l'orifice. Enfin, l'absence de fuite résiduelle était vérifiée en fin d'examen par une opacification au produit de contraste. Lors de chaque traitement endoscopique étaient relevés le diamètre de l'orifice fistuleux si celui-ci était persistant et visualisé en endoscopie $(<1 \mathrm{~cm}, 1-2 \mathrm{~cm},>2 \mathrm{~cm})$, l'aspect des berges de l'orifice (nécrose, issue de pus, collection abcèdée). On notait également le matériel endoscopique mis en place: pour les prothèses œsophagiennes: leur type (métalliques, complètement couvertes ou doubles types), leurs longueur et diamètre; pour les clips : leur type, leur nombre, éventuellement leur association à un traitement par prothèse.

Une endoscopie était systématiquement effectuée après 6 à 8 semaines maximum en l'absence de complications, permettant d'une part le retrait de la prothèse, et d'autre part l'évaluation de l'efficacité du traitement et de la nécessité d'un nouveau traitement endoscopique. A partir de cette deuxième endoscopie et lors de chaque endoscopie ultérieure étaient relevées la migration et l'extirpabilité du matériel endoscopique mis en place (seulement pour les prothèses), la persistance d'un orifice fistuleux ou d'un trajet fistuleux après retrait des prothèses (et/ou avec clips toujours en place) et par opacification. Si le premier traitement endoscopique ne permettait pas de fermer la fistule, alors il était répété jusqu'à obtention d'une efficacité, la décision d'une prise en charge chirurgicale (complication, échec), ou encore le décès du patient.

\section{Analyse statistique}

Les analyses statistiques ont été réalisées à l'aide du logiciel IBM SPSS Statistics version 20.0 (Inc., IL., USA). Les variables continues sont présentées sous forme de moyenne \pm écart-type ou sous forme de médiane avec le premier et troisième quartile. Les variables qualitatives sont présentées sous forme d'effectif et de pourcentages. Le lien entre variables qualitatives a été évalué à l'aide du test du chi deux de Pearson ou le test exact de Fisher si les effectifs théoriques étaient inférieurs à 5. La liaison entre une variable qualitative et une variable quantitative a été évaluée à l'aide du test T de Student ou le test non paramétrique de Mann Whitney. Les variables qui ont une $p$ valeur $<0,10$ en analyse univariée et celles qui ont une relevance clinique ont été introduites dans l'analyse multivariée. Le modèle de régression logistique a été utilisé pour identifier les facteurs prédictifs indépendants qui influent sur la réponse au traitement endoscopique. Le test d'Hosmer Lemeshow a été utilisé pour tester l'adéquation des données au modèle logistique. Pour tous les tests, la signification statistique est fixée à $p<0,05$. 


\section{RESULTATS}

\section{Caractéristiques des patients}

Au total, 126 patients ont été opérés pour un cancer de l'œsophage ou de la jonction œsocardiale par l'équipe de chirurgie thoracique de l'hôpital Nord à Marseille entre juin 2010 et juin 2014. Dans cette population, 68 patients ont été opérés selon la technique de Lewis-Santy, 44 selon la technique d'Akiyama et les 14 patients restant ont bénéficié d'autres types de chirurgie. De plus, 91 patients ont reçu une radio-chimiothérapie préopératoire et 30 patients ont été opérés sans traitement néo-adjuvant préalable (information manquante chez 5 patients). Le taux de complication post-opératoire liée à un lâchage anastomotique ou à une nécrose de l'anastomose (traité par voie endoscopique ou par reprise chirurgicale) a été de $29,4 \%$ dans le groupe Lewis-Santy et de $43,2 \%$ dans le groupe Akiyama $(p=0,135)$. Ce taux était de $34 \%$ dans le groupe radio-chimiothérapie et de $33,3 \%$ dans le groupe n'ayant pas reçu de traitement néo-adjuvant $(p=0,941)$. Au total, 68 patients $(53,97 \%)$ ont eu des suites opératoires simples, alors que 58 patients ont eu des complications postopératoires.

Parmi ces 58 patients (Figure1), 35 patients (27,78\%) ont eu une endoscopie dans les suites de la chirurgie confirmant une fistule ou un lâchage anastomotique et ont bénéficié d'un traitement endoscopique. Sur les 23 patients restant, trois (2,38\%) ont bénéficié d'une reprise chirurgicale sans endoscopie préalable (1 patient avec un chylothorax avec découverte d'une fistule digestive haute en per-opératoire, 1 patient ayant présenté un emphysème sous cutané à $\mathrm{J} 2$ de la chirurgie, 1 patient avec une fistule digestive haute vue au scanner). Quatre patients (3,17\%) ont eu une endoscopie digestive haute pour dégradation clinique et ont été réopérés car le traitement endoscopique était impossible ( 3 patients avec une nécrose gastrique complète, 1 patient avec de multiples trajets fistuleux anastomotiques complexes). Seize patients $(12,70 \%)$ ont eu une endoscopie digestive haute pour des indications diverses (méléna, sepsis) sans diagnostic de fistule retenu (7 endoscopies normales, 3 hémorragies digestives dont une massive avec décès, 5 zones de nécrose très peu étendues ne nécessitant pas de traitement spécifique, 1 ischémie gastrique majeure sans traitement endoscopique ni chirurgical possible).

Finalement, ce sont les 35 patients avec un diagnostic posé de fistule et qui ont bénéficié d'un traitement endoscopique qui ont été analysés (Tableau 1). Parmi eux, il y avait 4 femmes (11,4\%) et 31 hommes (88,6\%), l'âge moyen au diagnostic de fistule était de 61,7 ans $\pm 8,9$ [43-85]. 25 patients $(71,4 \%)$ avaient reçu un traitement par radio-chimiothérapie en préopératoire (traitement néo-adjuvant ou traitement exclusif suivi d'une chirurgie de rattrapage). Les techniques 
chirurgicales employées avaient été de type Lewis-Santy chez 17 (48,6\%) patients et Akiyama chez 16 autres (45,7\%). Une chirurgie selon la technique de Sweet avait été réalisée chez 1 patient, et une dégastro-gastrectomie chez 1 autre patient qui avait déjà bénéficié d'une chirurgie de type LewisSanty. Sur les pièces de résection ont été retrouvé 18 carcinomes épidermoïdes $(51,4 \%)$ et 17 adénocarcinomes (48,6\%). Les marges de résection étaient non saines chez 4 patients $(11,4 \%)$. Aucun patient ne présentait une maladie métastatique.

\section{Diagnostic et caractéristiques des fistules}

Le délai médian entre la réalisation de la chirurgie et le diagnostic de la fistule digestive haute était de 7 jours (IQR ; 5,00-11,25). La fistule était fortement suspectée au préalable sur un examen radiologique (scanner ou TOGD) chez 12 patients $(34,3 \%)$ alors que chez les 23 autres patients $(65,7 \%)$ le diagnostic a été posé par l'endoscopie digestive réalisée devant une dégradation clinique. D'autre part, 27 patients $(77,1 \%)$ étaient hospitalisés en service de réanimation au moment de la réalisation de la première endoscopie digestive.

Lors de la première endoscopie digestive, la hauteur moyenne de l'orifice fistuleux définie par sa distance par rapport aux arcades dentaires était de $23,75 \mathrm{~cm} \pm 4,6$ [16-35]. Le diamètre de l'orifice fistuleux était $<1 \mathrm{~cm}$ chez 14 patients (40\%), compris entre 1 et $2 \mathrm{~cm}$ chez 17 patients $(48,6 \%)$ et supérieur à $2 \mathrm{~cm}$ chez 4 patients $(11,4 \%)$. Les berges de l'orifice fistuleux étaient nécrosées chez 8 patients $(22,8 \%)$.

\section{Objectif principal : efficacité du traitement endoscopique}

L'efficacité primaire de la prise en charge endoscopique des fistules et lâchages anastomotiques post opératoires était de $48,6 \%$ (17 patients) avec un délai médian de guérison de 42 jours (IQR ; 28,5-52,5). L'efficacité secondaire et finale était de 68,6\% (24 patients) (Tableau 2). Chez les 7 patients ayant nécessité plusieurs reprises endoscopiques afin d'obtenir une efficacité du traitement, le délai médian de guérison a été de 67,5 jours (IQR ; 48,25-95,75). Sur les 11 patients $(31,4 \%)$ en échec thérapeutique, $6(17,1 \%)$ sont décédés (4 décès par syndrome de détresse respiratoire aiguë, 1 décès par choc septique, 1 décès par ischémie mésentérique) et $5(14,3 \%)$ ont bénéficié d'une reprise chirurgicale (4 reprises chirurgicales pour fistules œso-bronchiques persistantes, 1 reprise pour hémorragie digestive massive non accessible à un traitement endoscopique) avec un taux de mortalité de $40 \%$. 


\section{Objectifs secondaires:}

\section{- Délai du traitement endoscopique}

Le délai médian entre la réalisation de la chirurgie et la réalisation du premier traitement endoscopique était de 8,5 jours (IQR ; 6,00-18,25). Le délai médian entre la première et la deuxième endoscopie digestive était de 28,5 jours (IQR ; 16,75-36,25). 2 patients $(5,7 \%)$ sont décédés avant la réalisation de la deuxième endoscopie ( 1 syndrome de détresse respiratoire aigu, 1 ischémie mésentérique) et 1 patient (2,9\%) a bénéficié d'une reprise chirurgicale précoce sur hémorragie digestive massive non accessible à un traitement endoscopique. 18 patients $(58,1 \%)$ étaient toujours hospitalisés en réanimation au moment de la réalisation de la deuxième endoscopie digestive.

Le délai médian de guérison à partir du diagnostic initial de fistule était de 44 jours (IQR ; 33,75$72,25)$.

\section{- Caractéristiques du traitement endoscopique}

Lors du premier traitement endoscopique de ces fistules, 31 patients $(88,6 \%)$ ont bénéficié de la mise en place des prothèses œsophagiennes métalliques couvertes ou double-types. La longueur moyenne des prothèses était de $12,38 \mathrm{~cm} \pm 2,9$ [10-23]. Cinq patients (14,3\%) ont été traités par mise en place de clips ( 4 traitements par clips OVESCO, 1 traitement par clips standards) et 2 patients $(5,7 \%)$ ont bénéficié de la mise en place d'un drain "naso-fistule ". Parmi eux, 4 patients $(11,4 \%)$ ont bénéficié d'un traitement combiné (prothèse + clip).

Le nombre moyen d'endoscopies réalisées par patient a été de 2,6 $\pm 1,57$ [1-10]. Le nombre moyen de prothèses œsophagiennes mises en place par patient a été de 1,6 $\pm 1,35$ [0-7]. Un traitement combiné (associant différents types de traitement endoscopique) a été nécessaire chez 9 patients (26\%). II n'y avait pas de différence majeure entre le groupe Lewis-Santy et le groupe Akiyama en termes de nombre moyen d'endoscopies réalisées $(2,4$ versus 2,8$)$ et de prothèses mises en place $(1,65$ versus 1,62$)$. Chez les patients en efficacité secondaire, le nombre moyen d'endoscopies nécessaires a été 2,58 $\pm 0,93$ [2-5].

La durée médiane d'hospitalisation chez les patients en efficacité finale a été de 48,5 jours (IQR ; 33-77,5) et celle chez les 68 patients ayant eu des suites opératoires simples de 22 jours (IQR ; $16,5-27,5)(p<0,001)$.

\section{- Complications}

II n’y a eu aucun échec de mise en place de matériel endoscopique (prothèse, clip ou drain naso-fistule). 
Pour ce qui est de la première endoscopie, le taux d'extirpabilité des prothèses œsophagiennes était de $96 \%$ et le taux de migration était de $21,4 \%$. Devant une prothèse non extirpable était employée la technique de "stent in stent " consistant en la mise en place d'une prothèse couverte au sein de la première prothèse afin d'entrainer une nécrose de la muqueuse ayant bourgeonné à travers les mailles de la prothèse ou à ses extrémités. Puis les deux prothèses étaient retirées dans le même temps environ trois à quatre semaines plus tard. Pour ce qui est de la migration prothétique : si celle-ci était minime alors la prothèse était simplement repositionnée afin de bien couvrir le trajet fistuleux, si la migration était plus importante alors la prothèse était retirée définitivement et remplacée par une autre.

Le taux final d'extirpabilité était de 93,2\% (ramené à 100\% avec la technique du « stent in stent $»)$ et le taux de migration de $18 \%$ dont 8 étaient des prothèses métalliques totalement couvertes et 1 seule une prothèse métallique double-type. Un patient a présenté une hémorragie digestive sur une érosion muqueuse au pôle inférieur de sa prothèse œsophagienne avec nécessité de transfusion de culots globulaires, retrait de la prothèse et remplacement par deux nouvelles prothèses à but hémostatique et pour couvrir la fistule anastomotique persistante.

Le taux de mortalité en cas de traitement endoscopique a été de 17,1\% (6 patients sur 35) dont aucun décès ne pouvant être relié au traitement endoscopique lui-même (4 décès par syndrome de détresse respiratoire aigu, 1 décès par choc septique, 1 décès par ischémie mésentérique).

\section{Analyse univariée en sous-groupe pour l'efficacité du traitement}

- En fonction de la technique opératoire :

L'efficacité primaire du traitement endoscopique était de $47,1 \%$ dans le groupe de patients opérés selon la technique de Lewis-Santy (17 patients) et de 50\% dans le groupe de patients opérés selon la technique d'Akiyama (16 patients) $(p=0,866)$. L'efficacité secondaire était respectivement de $64,7 \%$ et de $68,75 \%(p=0,805)$. Le taux de reprise chirurgicale était de $18,75 \%$ dans le groupe Akiyama versus $11,8 \%$ dans le groupe Lewis-Santy $(p=0,656)$ et les taux de mortalité respectivement de $12,5 \%$ et $23,5 \% \quad(p=0,656)$. II n'y avait donc pas de différence statistiquement significative que ce soit en termes d'efficacité du traitement endoscopique, de taux de mortalité ou de reprise chirurgicale en fonction de la technique chirurgicale. Le délai médian de guérison était de 44 jours (IQR; 35,5-67,5) dans le groupe Lewis-Santy versus 43,5 jours (IQR; 39,75-93,5) dans le groupe Akiyama. 
- En fonction de la réalisation ou non d'une RCT néo-adjuvante

L'efficacité primaire du traitement endoscopique était de $48 \%$ dans le groupe de patients ayant reçu une radio-chimiothérapie pré-opératoire ( 25 patients) et de $50 \%$ dans le groupe n'en ayant pas reçu (10 patients) ( $p=0,99)$. L'efficacité secondaire était respectivement de $68 \%$ et $70 \%$ $(p=0,99)$. Le taux de mortalité était de $24 \%$ dans le groupe radio-chimiothérapie versus $0 \%(p=0,296)$ dans le groupe sans traitement néoadjuvant et les taux de reprise chirurgical étaient respectivement de $8 \%$ et $30 \%(p=0,128)$. Le délai médian de guérison était de 42 jours (IQR; 35,5-55,5) dans le groupe radio-chimiothérapie versus 52 jours dans I'autre groupe (IQR; 36,5-104). Aucune de ces différences ne s'est révélée significative.

\section{- En fonction de la largeur de l'orifice fistuleux}

L'efficacité primaire du traitement endoscopique était de $42,8 \%$ dans le groupe de patients dont le diamètre de la fistule est $<1 \mathrm{~cm}$, de $52,9 \%$ dans le groupe de patients dont le diamètre de la fistule est compris entre 1 et $2 \mathrm{~cm}$, et de $50 \%$ dans le groupe de patients dont le diamètre de la fistule est $>2 \mathrm{~cm}(p=0,89)$. L'efficacité secondaire était respectivement de $57,1 \%, 82,3 \%$ et $50 \%$ $(p=0,253)$. Le taux de reprise chirurgicale de $50 \%$ dans le groupe « $>2 \mathrm{~cm}$ » versus $14,3 \%$ et $5,9 \%$ dans les groupes $"<1 \mathrm{~cm}$ » et $\| 1 \mathrm{~cm}<\mathrm{t}<2 \mathrm{~cm} »(\mathrm{p}=0,091)$ et les taux de mortalité étaient respectivement de $0 \%$ versus $28,6 \%$ et $11,8 \%(p=0,44)$. Le délai médian de guérison était de 45 jours (IQR ; 35-52,5) dans le groupe de patients dont le diamètre de la fistule est $<1 \mathrm{~cm}$, de 44 jours (IQR ; $33,75-95,75)$ dans le groupe de patients dont le diamètre de la fistule est compris entre 1 et $2 \mathrm{~cm}$, et de 42 jours (IQR ; 42-42) dans le groupe de patients dont le diamètre de la fistule est $>2 \mathrm{~cm}$.

- En fonction du délai entre la chirurgie, le diagnostic de fistule et la prise en charge de celle-ci

Le délai médian entre la chirurgie et le diagnostic de fistule était de 9 jours (IQR ; 6-17) dans le groupe de patients en efficacité primaire et de 7 jours (IQR ; 5-8) dans le groupe de patients en échec primaire $(p=0,183)$. Le délai médian entre la chirurgie et le diagnostic de fistule était de 7 jours (IQR ; 6-12) dans le groupe en efficacité secondaire et de 7 jours (IQR ; 4,5-9,5) dans le groupe en échec final $(p=0,405)$. Le délai médian entre la chirurgie et le premier traitement endoscopique de la fistule était de 12,5 jours (IQR ; 6-21,25) dans le groupe en efficacité primaire et de 8 jours (IQR ; 511) dans le groupe en échec primaire $(p=0,125)$. Le délai médian entre la chirurgie et le premier traitement endoscopique de la fistule était de 10 jours (IQR ; 6-18,25) dans le groupe en efficacité secondaire et de 8 jours (IQR ; 4,5-10,5) dans le groupe en échec final $(p=0,301)$.

Il n'y avait pas d'association significative entre le fait que le patient ait été hospitalisé en service de réanimation au moment du premier traitement endoscopique et le taux d'efficacité 
primaire du traitement endoscopique $(p=0,443)$, ni avec le taux d'efficacité secondaire de ce traitement $(p=0,387)$. II n'y avait pas non plus de lien statistiquement significatif entre l'âge moyen au diagnostic de fistule et le taux d'efficacité primaire du traitement endoscopique $(p=0,101)$. II en était de même avec le taux d'efficacité secondaire $(p=0,165)$.

\section{Analyses multivariée pour l'efficacité du traitement}

L'analyse multivariée associant la taille de la fistule, le type d'intervention chirurgicale et la présence ou non d'une radiochimiothérapie néo adjuvante n'a pas montré d'association significative entre ces trois critères et l'efficacité primaire et finale du traitement endoscopique.

\section{DISCUSSION}

Les fistules anastomotiques post-œsophagectomie pour cancer de l'œsophage ou de la jonction œso-gastrique (JOG) sont une complication grave de cette chirurgie dont la fréquence varie de 5 à $20 \%$ en fonction des séries mais dont le taux de mortalité serait de $40 \%$ en postopératoire $(9,10)$. Depuis une dizaine d'années, le traitement endoscopique de ces fistules semble s'imposer comme le nouveau standard de prise en charge face au traitement conservateur ou à la reprise chirurgicale qui s'accompagnent de taux de mortalité variant de 40 à $100 \%(9,10,12)$. Or à ce jour, relativement peu d'études avaient correctement évalué son efficacité et ses résultats, avec une majorité de petite série de cas (16-32). Notre travail, qui a permis d'étudier rétrospectivement l'efficacité du traitement endoscopique chez 35 patients opérés l'hôpital Nord à Marseille pour cancer de l'œsophage ou de la JOG entre 2010 et 2014 et qui ont présenté une fistule anastomotique en post-opératoire, est à notre connaissance la plus importante série dans la littérature.

Concernant la stratégie thérapeutique, le matériel endoscopique utilisé a été pour la grande majorité des prothèses métalliques couvertes ou double-types et pour quelques cas des clips larges OVESCO $^{\odot}$ (Ovesco, Tubingen, Allemagne), des drains naso-fistules voire un traitement combiné. Dans notre étude, l'efficacité primaire était de $48,6 \%$ et l'efficacité finale de $68,6 \%$. Ces résultats sont légèrement inférieurs à ceux retrouvés dans la littérature, mais probablement plus proches de la réalité du fait de la grande taille de notre série et de l'inclusion initiale de tous les patients consécutivement opérés sur cette période. Différents types de prothèses ont été utilisés dans cette indication. Pour ce qui est des prothèses plastiques, leur efficacité finale variait de 60 à $95 \%$ selon les cas $(16,17,21,23-26)$. L'efficacité des prothèses métalliques allait, elle, de 77 à $100 \%$ pour les totalement couvertes $(20,23,27-32)$ et de 72 à $73 \%$ pour les partiellement couvertes $(22,23)$. Cette 
différence peut s'expliquer d'une part par le fait que notre étude soit l'une des seules à présenter de manière exhaustive en intention de traiter tous les patients ayant présenté une fistule en postœsophagectomie pour cancer sur une période de 4 ans alors que certaines séries présentent des case-séries triés et sélectionnés parmi leur expérience endoscopique parce qu'ils ont obtenu une guérison, omettant certains échecs. D’autre part, ces études représentent une population très hétérogène en ce qui concerne l'indication du traitement endoscopique (fistule après œsophagectomie pour cancer de l'œsophage, après gastrectomie, après chirurgie bariatrique, plus rarement syndrome de Boerhaave ou perforation iatrogène). En effet, seulement 2 études portent strictement sur les fistules anastomotiques post-œsophagectomie pour cancer de l'œsophage avec une efficacité variant de 77 à $89 \%(18,24)$ mais avec des effectifs plus faibles (9 et 22 patients respectivement). De plus, $77,1 \%$ de nos patients étaient hospitalisés en réanimation au moment du premier traitement endoscopique soulignant leur gravité clinique pouvant avoir impacté sur l'efficacité finale par décès précoce ou nécessité de reprise chirurgicale rapide. Au final, nos résultats en terme d'efficacité primaire et secondaire sont assez proches de ceux obtenus lors du traitement endoscopique des fistules après chirurgie bariatrique $(13,22,33)$.

Le taux de migration prothétique retrouvé dans notre série s'élevait à $18 \%$ sans aucun décès ni complication grave lié aux prothèses. Dans la littérature, les taux de migration vont respectivement de 17 à $45 \%$ pour les prothèses plastiques $(16,17,23,25,26)$, de 10 à $42 \%$ pour les prothèses métalliques entièrement couvertes $(20,23,28-30,32)$ et de 10 à $16 \%$ pour les prothèses métalliques double-types $(22,23)$. Sur les 9 migrations observées parmi nos patients, 8 ont eu lieu avec des prothèses métalliques entièrement couvertes et seulement une avec une prothèse doubletype, soulignant l'intérêt de ce type de prothèse dans la prise en charge thérapeutique des pathologies fistuleuses comme cela a été déjà montré dans l'étude de Gonzalez et al.(22). Parallèlement à cela, le taux d'extirpabilité des prothèses a été amené de 93 à $100 \%$ grâce à la technique de "stent-in-stent » qui s'est déjà révélée efficace dans plusieurs séries publiées (34-36). Les 3 prothèses métalliques entièrement couvertes initialement non extirpables ont pu être retirées grâce à cette technique avec nécessité de deux tentatives pour l'une des prothèses. A noter que de par leur taux de migration élevé pouvant entrainer d'autres complications à type de perforation digestive $(37,38)$, et/ou de persistance de la fistule, les prothèses œsophagiennes plastiques ne sont plus utilisées dans notre service d'endoscopie. Enfin, sur les 8 clips OVESCO mis en place, cinq ont dû être associés à une prothèse devant la persistance d'un trajet fistuleux à l'opacification et sur les 3 clips employés en monothérapie, un seul a permis d'obtenir une efficacité finale. Selon notre expérience, l'emploi des clips OVESCO doit rester une alternative de $2^{\text {ème }}$ intention dans cette indication, et doit être réservé aux fistules $<1 \mathrm{~cm}$ drainées et en association à une prothèse. 
Parmi les critères d'évaluation secondaires de notre étude, nous avons d'abord cherché à déterminer l'influence des deux principales techniques chirurgicales employées sur l'efficacité du traitement endoscopique. Dix-sept patients ont été opérés selon la technique de Lewis-Santy qui associe une laparotomie et une thoracotomie droite avec une anastomose thoracique et 16 patients selon la technique d'Akiyama qui associe une troisième voie d'abord par cervicotomie gauche avec une anastomose cervicale. Historiquement, les anastomoses cervicales se compliquent plus fréquemment de fistules que les anastomoses thoraciques mais sont associées à un taux de mortalité plus bas (10,39-42). En effet, leur drainage pourrait être amélioré par réouverture de la cicatrice cervicale. Sur les 126 patients opérés pour cancer de l'œsophage ou de la JOG dans notre centre sur 4 ans, on notait une tendance en terme de fréquence dans le groupe Akiyama avec $43,2 \%$ de complications à type de fistule ou de nécrose de l'anastomose versus $29,4 \%$ dans le groupe LewisSanty $(p=0,135)$ ce qui est concordant avec la littérature. Sur les 35 patients étudiés, il n'y avait pas de différence significative en terme d'efficacité primaire et finale du traitement endoscopique, ni en terme de mortalité, de taux de reprise chirurgicale, ou encore de délai de guérison en fonction du type de chirurgie. Ces résultats, malgré notre effectif peut-être insuffisant, sont également retrouvés dans d'autres études. Dans l'article de Blewett et $a l$, une analyse rétrospective de 74 patients opérés pour cancer de l'œsophage ne montrait pas de différence significative en terme de taux de fréquence des fistules en fonction de la localisation cervicale ou thoracique de l'anastomose $(p=0,21)$ ni en terme de taux de mortalité consécutive à la fistule entre les deux groupes $(p=0,74)(43)$. De même, Lam et al. soulignaient l'absence de différence en termes de fréquence des fistules anastomotiques entre les 2 types d'anastomoses (44). Enfin dans l'étude d'Alanezi et al, 23 patients ont présenté une fistule en post-œsophagectomie avec une différence non significative $(p=0,91)$ en ce qui concerne le taux de mortalité liée à la fistule entre les deux groupes (9). Aux vues des données de la littérature et à la lumière de nos résultats, nous n'avons pas de recommandation spécifique à faire aux chirurgiens concernant le type d'anastomose qui est à réaliser en fonction de la localisation néoplasique. Le choix du chirurgien devrait donc se porter sur la technique chirurgicale qu'il maîtrise le mieux, du moins en attendant d'autres études plus larges.

D'autre part, l'impact d'un traitement pré-opératoire par radiochimiothérapie (RCT) sur les taux de morbidité et de mortalité post-opératoires est un sujet d'inquiétude pour les équipes chirurgicales. Dans la méta-analyse de Fiorica et $a l$, les résultats combinés de 6 essais contrôlés randomisés lient significativement la RCT à une augmentation du taux de mortalité post-opératoire (OR 2,10 [95\% Cl 1,18-3,73]; $p=0,01$ ) (45). Deux autres essais ont associé la RCT avec une augmentation du taux de complications post-opératoires parmi lesquelles les fistules anastomotiques $(46,47)$. Dans l'étude de D'journo et al, portant sur les œsophagectomies de 
rattrapage, l'incidence des fistules anastomotiques était significativement augmentée par une dose d'irradiation dépassant 55 Grays (5). Ces résultats n'ont pas été retrouvés dans notre série sur les 126 patients opérés dans notre centre, puisque le taux de fistule anastomotique était quasiment identique entre le groupe de patients ayant reçu une RCT pré-opératoire (les doses reçues n'ont pu être récupérées) et le groupe de patients opérés d'emblée $(p=0,941)$. Parmi les 35 patients étudiés, on note une tendance quant à une augmentation du taux de mortalité dans le groupe RCT ( $24 \%$ versus $0 \%)$ sans pour autant que ce résultat soit significatif $(p=0,296)$, probablement par manque de puissance. En revanche, on ne retrouve encore aucune différence en termes d'efficacité primaire et finale du traitement endoscopique, ni concernant le taux de reprise chirurgicale ou le délai de guérison. Nos résultats semblent être en accord avec d'autres études comme celle de JunemannRamirez et al. qui suggère que la RCT n'est pas un facteur de risque de fistule post-œsophagectomie (48). De même, chez Schweigert et al. on retrouve la même fréquence de RCT pré-opératoire entre le groupe de patients ayant fistulisé et ceux n'ayant pas présenté de complication anastomotique $(p=0,36)(18)$. L'essai de Martin et al. qui comparait de façon rétrospective l'incidence des fistules anastomotiques entre la période 1970-1986 et entre la période 1987-2004, a montré une stabilité de l'incidence malgré une nette augmentation de l'utilisation de la RCT pré-opératoire entre ces deux périodes (49). Enfin chez Fiorica et al, le taux de complications post-opératoires (incluant complications respiratoires, fistules anastomotiques et insuffisance cardiaque) n'était pas significativement différent entre les deux groupes $(p=0,16)(45)$. S'il est encore difficile d'affirmer que la radio-chimiothérapie préopératoire est un facteur de risque de fistule anastomotique postœsophagectomie, cette complication semble répondre au traitement endoscopique avec la même efficacité sur tissus irradiés ou non.

Pour ce qui est de la taille de l'orifice fistuleux, elle ne semble pas influer, probablement par manque de puissance, sur l'efficacité du traitement endoscopique même s'il faut souligner que les fistules de plus de $2 \mathrm{~cm}$ de diamètre ont tendance à bénéficier plus souvent d'une reprise chirurgicale que les autres $(p=0,091)$. Notre étude semble être la seule à avoir recherché l'influence de ce facteur sur les résultats de la prise en charge endoscopique dans cette indication. Au final, que ce soit en analyse uni ou multivariée, nous n'avons pas mis en évidence de manière significative de facteur influant sur l'efficacité du traitement endoscopique des fistules anastomotiques postœsophagectomie pour cancer de l'œsophage ou de la jonction œso-gastrique. Concernant les délais de traitement, notre délai de prise en charge endoscopique fut court, avec un délai médian de 8,5 jours entre la chirurgie et le premier traitement endoscopique, assurant une réponse rapide aux demandes des réanimateurs et chirurgiens. Le délai médian entre la $1^{\text {ère }}$ et la $2^{\text {ème }}$ endoscopie a été de 28,5 jours, durée offrant le meilleur rapport efficacité/extirpabilité des stents métalliques. En 
effet, même s'il n'y a à ce jour pas de recommandations indiquant la durée optimale à partir de laquelle il est licite de vérifier l'étanchéité du montage chirurgical, des études animales ont suggéré que 30 jours seraient suffisants pour permettre une cicatrisation (50).

Le taux de mortalité dans notre groupe de patients traités par voie endoscopique a été de $17,1 \%$ en comparaison au taux de $40 \%$ chez les patients repris chirurgicalement, ce qui souligne l'importance de la possibilité d'une prise en charge endoscopique première et l'extrême gravité clinique des patients bénéficiant d'une reprise chirurgicale qui constitue leur dernière chance. Les fistules anastomotiques représentent de plus une complication ayant un coût financier important avec une nette augmentation de la durée médiane d'hospitalisation par rapport aux patients ayant eu des suites opératoires simples $(48,5$ jours versus 22 jours; $p<0,001)$, mais cette augmentation s'effectue néanmoins au prix de la guérison du patient dans la majorité des cas. II faut souligner que le taux de fistule anastomotique post-œsophagectomie pour cancer de l'œsophage ou de la JOG a été particulièrement élevé dans notre centre avec un taux de $27,78 \%$. Selon les guidelines publiées en 2002 , l'incidence des fistules anastomotiques post-œsophagectomie ne devrait pas dépasser 5\% dans les centres experts (51).

Les limites de notre étude sont son caractère rétrospectif, unicentrique et son faible effectif. Il s'agit pourtant du plus gros effectif publié pour le moment et le recueil de données devrait se poursuivre dans les années à venir afin de voir peut-être émerger un jour un facteur influant sur l'efficacité du traitement endoscopique dans cette indication. En effet nous n'avons pas étudié le rôle de certains marqueurs dont ceux de la dénutrition (albumine notamment), ni l'implication des scores pronostiques à l'entrée en réanimation.

\section{CONCLUSION}

En conclusion, notre travail démontre l'efficacité du traitement endoscopique, en particulier des prothèses œsophagiennes métalliques, chez les patients présentant une fistule anastomotique post-œsophagectomie pour cancer de l'œsophage ou de la jonction œsogastrique quel que soit le type d'anastomose (cervicale ou thoracique) avec une efficacité primaire d'environ $50 \%$ et secondaire de presque $70 \%$. Ce traitement, lorsqu'il est possible, semble supérieur à la reprise chirurgicale en première intention même si celle-ci ne peut être parfois évitée. De plus, la migration des prothèses, complication principale de ce traitement, pourrait être minorée par l'utilisation des prothèses double-types dans cette indication. 


\section{Références}

1. Ferlay J, Shin H-R, Bray F, Forman D, Mathers C, Parkin DM. Estimates of worldwide burden of cancer in 2008: GLOBOCAN 2008. Int J Cancer J Int Cancer. 15 déc 2010;127(12):2893-917.

2. Zhang Y. Epidemiology of esophageal cancer. World J Gastroenterol WJG. 14 sept 2013;19(34):5598-606.

3. Chennat J, Waxman I. Endoscopic treatment of Barrett's esophagus: From metaplasia to intramucosal carcinoma. World J Gastroenterol WJG. 14 août 2010;16(30):3780-5.

4. Bedenne L, Michel P, Bouché O, Milan C, Mariette C, Conroy T, et al. Chemoradiation followed by surgery compared with chemoradiation alone in squamous cancer of the esophagus: FFCD 9102. J Clin Oncol Off J Am Soc Clin Oncol. 1 avr 2007;25(10):1160-8.

5. D'Journo X-B, Michelet P, Dahan L, Doddoli C, Seitz J-F, Giudicelli R, et al. Indications and outcome of salvage surgery for oesophageal cancer. Eur J Cardio-Thorac Surg Off J Eur Assoc Cardio-Thorac Surg. juin 2008;33(6):1117-23.

6. Barreto JC, Posner MC. Transhiatal versus transthoracic esophagectomy for esophageal cancer. World J Gastroenterol WJG. 14 août 2010;16(30):3804-10.

7. Hulscher JBF, van Sandick JW, de Boer AGEM, Wijnhoven BPL, Tijssen JGP, Fockens P, et al. Extended transthoracic resection compared with limited transhiatal resection for adenocarcinoma of the esophagus. N Engl J Med. 21 nov 2002;347(21):1662-9.

8. Walther B, Johansson J, Johnsson F, Von Holstein CS, Zilling T. Cervical or thoracic anastomosis after esophageal resection and gastric tube reconstruction: a prospective randomized trial comparing sutured neck anastomosis with stapled intrathoracic anastomosis. Ann Surg. déc 2003;238(6):803-12; discussion 812-4.

9. Alanezi K, Urschel JD. Mortality secondary to esophageal anastomotic leak. Ann Thorac Cardiovasc Surg Off J Assoc Thorac Cardiovasc Surg Asia. avr 2004;10(2):71-5.

10. Urschel JD. Esophagogastrostomy anastomotic leaks complicating esophagectomy: a review. Am J Surg. juin 1995;169(6):634-40.

11. Kusano C, Baba M, Takao S, Sane S, Shimada M, Shirao K, et al. Oxygen delivery as a factor in the development of fatal postoperative complications after oesophagectomy. Br J Surg. févr 1997;84(2):252-7.

12. Dai YY, Gretschel S, Dudeck O, Rau B, Schlag PM, Hünerbein M. Treatment of oesophageal anastomotic leaks by temporary stenting with self-expanding plastic stents. Br J Surg. août 2009;96(8):887-91.

13. Mercky P, Gonzalez J-M, Aimore Bonin E, Emungania O, Brunet J, Grimaud J-C, et al. Usefulness of over-the-scope clipping system for closing digestive fistulas. Dig Endosc Off J Jpn Gastroenterol Endosc Soc. janv 2015;27(1):18-24.

14. Fischer A, Höppner J, Utzolino S, Richter-Schrag H-J. Over-the-scope clip (OTSC) closure of a gastrobronchial fistula after esophagectomy. Endoscopy. 2014;46 Suppl 1 UCTN:E638-9. 
15. Morgan RA, Ellul JP, Denton ER, Glynos M, Mason RC, Adam A. Malignant esophageal fistulas and perforations: management with plastic-covered metallic endoprostheses. Radiology. août 1997;204(2):527-32.

16. Langer FB, Wenzl E, Prager G, Salat A, Miholic J, Mang T, et al. Management of postoperative esophageal leaks with the Polyflex self-expanding covered plastic stent. Ann Thorac Surg. févr 2005;79(2):398-403; discussion 404.

17. Schubert D, Scheidbach H, Kuhn R, Wex C, Weiss G, Eder F, et al. Endoscopic treatment of thoracic esophageal anastomotic leaks by using silicone-covered, self-expanding polyester stents. Gastrointest Endosc. juin 2005;61(7):891-6.

18. Schweigert M, Solymosi N, Dubecz A, Stadlhuber RJ, Muschweck H, Ofner D, et al. Endoscopic stent insertion for anastomotic leakage following oesophagectomy. Ann R Coll Surg Engl. janv 2013;95(1):43-7.

19. Brams A, Bulois P, Maunoury V, Triboulet J-P, Mariette C. Traitement des fistules anastomotiques intrathoraciques après œsophagectomie par prothèse couverte autoexpansive extractible. Gastroentérologie Clin Biol. janv 2008;32(1):41-5.

20. Zisis C, Guillin A, Heyries L, Lienne P, D'Journo X-B, Doddoli C, et al. Stent placement in the management of oesophageal leaks. Eur J Cardio-Thorac Surg Off J Eur Assoc Cardio-Thorac Surg. mars 2008;33(3):451-6.

21. Gutiérrez-Salmeán G, Peláez-Luna M, González-Galeote E, Lozoya-González D, Fuchs-Tarlovsky V, Farca-Belsaguy A. Outcomes of temporary self-expanding plastic stents (SEPS) use for gastrointestinal leaks. A case series. Rev Gastroenterol México. 2009;74(3):181-6.

22. Gonzalez J-M, Garces Duran R, Vanbiervliet G, Lestelle V, Gomercic C, Gasmi M, et al. Doubletype metallic stents efficacy for the management of post-operative fistulas, leakages, and perforations of the upper gastrointestinal tract. Surg Endosc. 11 oct 2014;

23. Van Boeckel PGA, Dua KS, Weusten BLAM, Schmits RJH, Surapaneni N, Timmer R, et al. Fully covered self-expandable metal stents (SEMS), partially covered SEMS and self-expandable plastic stents for the treatment of benign esophageal ruptures and anastomotic leaks. BMC Gastroenterol. 2012;12:19.

24. Hünerbein M, Stroszczynski C, Moesta KT, Schlag PM. Treatment of thoracic anastomotic leaks after esophagectomy with self-expanding plastic stents. Ann Surg. nov 2004;240(5):801-7.

25. Gelbmann CM, Ratiu NL, Rath HC, Rogler G, Lock G, Schölmerich J, et al. Use of self-expandable plastic stents for the treatment of esophageal perforations and symptomatic anastomotic leaks. Endoscopy. août 2004;36(8):695-9.

26. Freeman RK, Ascioti AJ, Wozniak TC. Postoperative esophageal leak management with the Polyflex esophageal stent. J Thorac Cardiovasc Surg. févr 2007;133(2):333-8.

27. Doniec JM, Schniewind B, Kahlke V, Kremer B, Grimm H. Therapy of anastomotic leaks by means of covered self-expanding metallic stents after esophagogastrectomy. Endoscopy. août 2003;35(8):652-8. 
28. Swinnen J, Eisendrath P, Rigaux J, Kahegeshe L, Lemmers A, Le Moine O, et al. Self-expandable metal stents for the treatment of benign upper GI leaks and perforations. Gastrointest Endosc. mai 2011;73(5):890-9.

29. Kauer WKH, Stein HJ, Dittler H-J, Siewert JR. Stent implantation as a treatment option in patients with thoracic anastomotic leaks after esophagectomy. Surg Endosc. janv 2008;22(1):50 $-3$.

30. Babor R, Talbot M, Tyndal A. Treatment of upper gastrointestinal leaks with a removable, covered, self-expanding metallic stent. Surg Laparosc Endosc Percutan Tech. févr 2009;19(1):e1 -4 .

31. Roy-Choudhury SH, Nicholson AA, Wedgwood KR, Mannion RA, Sedman PC, Royston CM, et al. Symptomatic malignant gastroesophageal anastomotic leak: management with covered metallic esophageal stents. AJR Am J Roentgenol. janv 2001;176(1):161-5.

32. Salminen $P$, Gullichsen $R$, Laine $S$. Use of self-expandable metal stents for the treatment of esophageal perforations and anastomotic leaks. Surg Endosc. juill 2009;23(7):1526-30.

33. Surace M, Mercky P, Demarquay J-F, Gonzalez J-M, Dumas R, Ah-Soune P, et al. Endoscopic management of GI fistulae with the over-the-scope clip system (with video). Gastrointest Endosc. déc 2011;74(6):1416-9.

34. Hirdes MMC, Siersema PD, Houben MHMG, Weusten BL a. M, Vleggaar FP. Stent-in-stent technique for removal of embedded esophageal self-expanding metal stents. Am J Gastroenterol. févr 2011;106(2):286-93.

35. Yang D-H, Seo M, Lee HJ, Park SH, Kim K-J, Ye BD, et al. Stent-in-stent technique and endoscopic resection of granulation tissue to remove a migrated metal duodenal stent embedded in the colon. Endoscopy. 2014;46 Suppl 1 UCTN:E159-60.

36. Langer FB, Schoppmann SF, Prager G, Riegler FM, Zacherl J. Solving the problem of difficult stent removal due to tissue ingrowth in partially uncovered esophageal self-expanding metal stents. Ann Thorac Surg. mai 2010;89(5):1691-2.

37. Eickhoff A, Knoll M, Jakobs R, Weickert U, Hartmann D, Schilling D, et al. Self-expanding metal stents versus plastic prostheses in the palliation of malignant dysphagia: long-term outcome of 153 consecutive patients. J Clin Gastroenterol. déc 2005;39(10):877-85.

38. Conio M, Repici A, Battaglia G, De Pretis G, Ghezzo L, Bittinger M, et al. A randomized prospective comparison of self-expandable plastic stents and partially covered self-expandable metal stents in the palliation of malignant esophageal dysphagia. Am J Gastroenterol. déc 2007;102(12):2667-77.

39. Valverde A, Hay JM, Fingerhut A, Elhadad A. Manual versus mechanical esophagogastric anastomosis after resection for carcinoma: a controlled trial. French Associations for Surgical Research. Surgery. sept 1996;120(3):476-83.

40. Müller JM, Erasmi $H$, Stelzner M, Zieren U, Pichlmaier H. Surgical therapy of oesophageal carcinoma. Br J Surg. août 1990;77(8):845-57. 
41. Whooley BP, Law S, Alexandrou A, Murthy SC, Wong J. Critical appraisal of the significance of intrathoracic anastomotic leakage after esophagectomy for cancer. Am J Surg. mars 2001;181(3):198-203.

42. Patil PK, Patel SG, Mistry RC, Deshpande RK, Desai PB. Cancer of the esophagus: esophagogastric anastomotic leak--a retrospective study of predisposing factors. J Surg Oncol. mars 1992;49(3):163-7.

43. Blewett CJ, Miller JD, Young JE, Bennett WF, Urschel JD. Anastomotic leaks after esophagectomy for esophageal cancer: a comparison of thoracic and cervical anastomoses. Ann Thorac Cardiovasc Surg Off J Assoc Thorac Cardiovasc Surg Asia. avr 2001;7(2):75-8.

44. Lam TC, Fok M, Cheng SW, Wong J. Anastomotic complications after esophagectomy for cancer. A comparison of neck and chest anastomoses. J Thorac Cardiovasc Surg. août 1992;104(2):395400.

45. Fiorica F, Di Bona D, Schepis F, Licata A, Shahied L, Venturi A, et al. Preoperative chemoradiotherapy for oesophageal cancer: a systematic review and meta-analysis. Gut. juill 2004;53(7):925-30.

46. Doty JR, Salazar JD, Forastiere AA, Heath El, Kleinberg L, Heitmiller RF. Postesophagectomy morbidity, mortality, and length of hospital stay after preoperative chemoradiation therapy. Ann Thorac Surg. juill 2002;74(1):227-31; discussion 231.

47. Zacherl J, Sendler A, Stein HJ, Ott K, Feith M, Jakesz R, et al. Current status of neoadjuvant therapy for adenocarcinoma of the distal esophagus. World J Surg. sept 2003;27(9):1067-74.

48. Junemann-Ramirez M, Awan MY, Khan ZM, Rahamim JS. Anastomotic leakage postesophagogastrectomy for esophageal carcinoma: retrospective analysis of predictive factors, management and influence on longterm survival in a high volume centre. Eur J Cardio-Thorac Surg Off J Eur Assoc Cardio-Thorac Surg. janv 2005;27(1):3-7.

49. Martin LW, Swisher SG, Hofstetter W, Correa AM, Mehran RJ, Rice DC, et al. Intrathoracic leaks following esophagectomy are no longer associated with increased mortality. Ann Surg. sept 2005;242(3):392-9; discussion 399-402.

50. Takimoto Y, Nakamura T, Yamamoto Y, Kiyotani T, Teramachi M, Shimizu Y. The experimental replacement of a cervical esophageal segment with an artificial prosthesis with the use of collagen matrix and a silicone stent. J Thorac Cardiovasc Surg. juill 1998;116(1):98-106.

51. Allum WH, Griffin SM, Watson A, Colin-Jones D, Association of Upper Gastrointestinal Surgeons of Great Britain and Ireland, British Society of Gastroenterology, et al. Guidelines for the management of oesophageal and gastric cancer. Gut. juin 2002;50 Suppl 5:v1-23. 


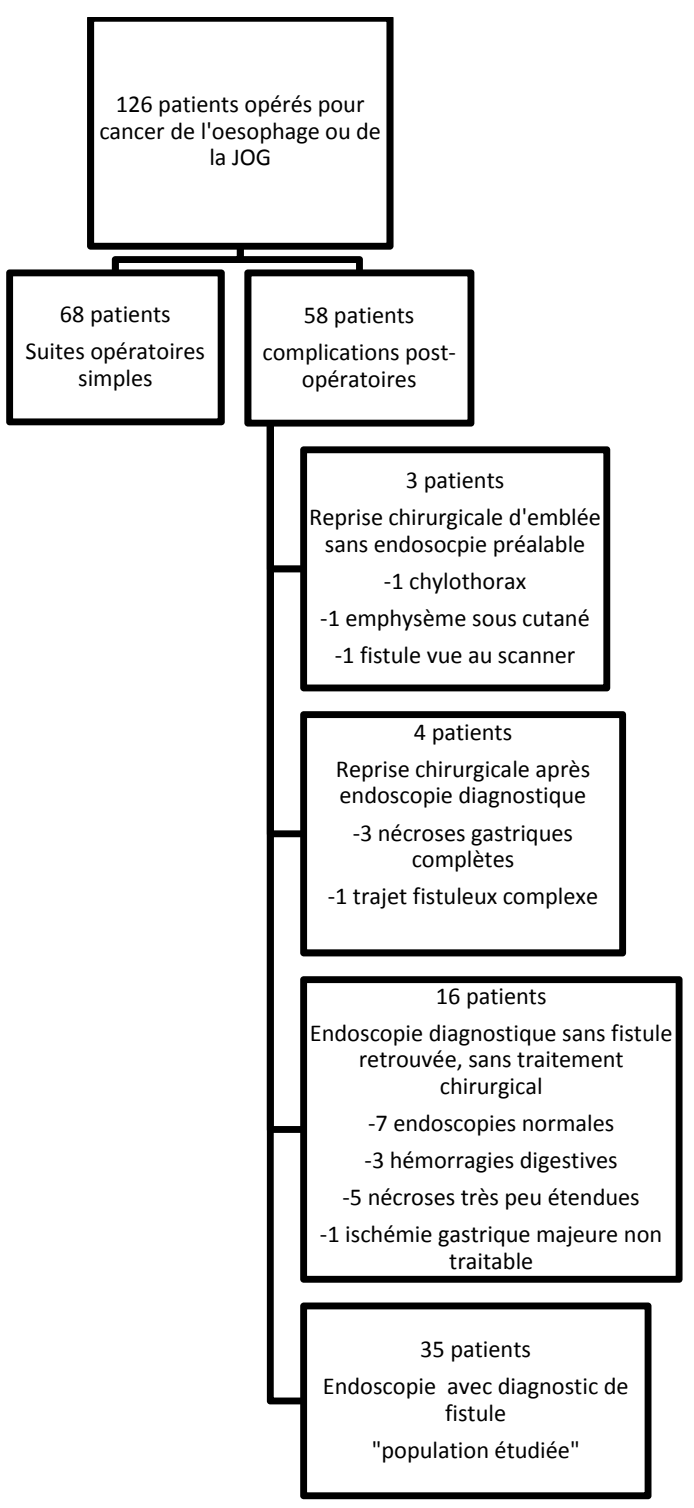

Figure 1 


\begin{tabular}{|c|c|c|}
\hline Caractéristiques des 35 patients & $\mathrm{n}$ & $\%$ \\
\hline \multicolumn{3}{|l|}{ Sexe } \\
\hline - Femmes & 4 & 11,4 \\
\hline - Hommes & 31 & 88,6 \\
\hline Age moyen au diagnostic de fistule (en années) & 61,7 & NA \\
\hline \multicolumn{3}{|l|}{ Radio-chimiothérapie pré-opératoire } \\
\hline -Oui & 25 & 71,4 \\
\hline- Non & 10 & 28,6 \\
\hline \multicolumn{3}{|l|}{ Technique chirurgicale } \\
\hline - Lewis-Santy & 17 & 48,6 \\
\hline - Akiyama & 16 & 45,7 \\
\hline - Sweet & 1 & 2,85 \\
\hline - Dégastro-gastrectomie & 1 & 2,85 \\
\hline \multicolumn{3}{|l|}{ Type histologique } \\
\hline - carcinome épidermoïde & 18 & 51,4 \\
\hline - adénocarcinome & 17 & 48,6 \\
\hline \multicolumn{3}{|l|}{ Qualité de résection } \\
\hline- RO & 31 & 88,6 \\
\hline$-R 1$ & 4 & 11,4 \\
\hline \multicolumn{3}{|l|}{ Moyen diagnostique de fistule } \\
\hline - Imagerie & 12 & 34,3 \\
\hline - Endoscopie & 23 & 65,7 \\
\hline \multicolumn{3}{|l|}{ Hospitalisation en réanimation } \\
\hline - Oui & 27 & 77,1 \\
\hline - Non & 8 & 22,9 \\
\hline $\begin{array}{l}\text { Hauteur moyenne de l'orifice fistuleux par } \\
\text { rapport aux arcades dentaires (en } \mathrm{cm} \text { ) }\end{array}$ & 23,75 & NA \\
\hline \multicolumn{3}{|l|}{ Diamètre de l'orifice fistuleux } \\
\hline$-<1 \mathrm{~cm}$ & 14 & 40 \\
\hline$-1 \mathrm{~cm} \leq \mathrm{t}<2 \mathrm{~cm}$ & 17 & 48,6 \\
\hline$-\geq 2 \mathrm{~cm}$ & 4 & 11,4 \\
\hline $\begin{array}{l}\text { Délai médian de prise en charge endoscopique } \\
\text { (jours) }\end{array}$ & 8,5 & NA \\
\hline
\end{tabular}

\section{Tableau 1}




\begin{tabular}{|l|c|c|c|c|c|c|c|c|c|c|c|}
\hline & Tous & \multicolumn{2}{|c|}{ Type de chirurgie } & \multicolumn{2}{|c|}{ Traitement néo-adjuvant } & \multicolumn{3}{|c|}{ Taille de la fistule } \\
\hline & $\begin{array}{c}\text { Lewis- } \\
\text { Santy } \\
\mathrm{n}=17\end{array}$ & $\begin{array}{c}\text { Aki- } \\
\text { yama } \\
\mathrm{n}=16\end{array}$ & $p$ & $\begin{array}{c}\mathrm{RTCT} \\
\mathrm{n}=25\end{array}$ & $\begin{array}{c}\text { Pas } \\
\mathrm{RTCT} \\
\mathrm{n}=10\end{array}$ & $p$ & $\begin{array}{c}\mathrm{T}<1 \mathrm{~cm} \\
\mathrm{n}=14\end{array}$ & $\begin{array}{c}1 \leq \mathrm{T}<2 \mathrm{~cm} \\
\mathrm{n}=17\end{array}$ & $\begin{array}{c}\mathrm{T} \geq 2 \mathrm{~cm} \\
\mathrm{n}=4\end{array}$ & $p$ \\
\hline $\begin{array}{l}\text { Efficacité } \\
\text { primaire }\end{array}$ & $\mathbf{4 8 , 6 \%}$ & $\begin{array}{c}47,1 \% \\
\mathrm{n}=8\end{array}$ & $\begin{array}{c}50 \% \\
\mathrm{n}=8\end{array}$ & 0,86 & $\begin{array}{c}48 \% \\
\mathrm{n}=12\end{array}$ & $\begin{array}{c}50 \% \\
\mathrm{n}=5\end{array}$ & 0,99 & $\begin{array}{c}42,8 \% \\
\mathrm{n}=6\end{array}$ & $\begin{array}{c}52,9 \% \\
\mathrm{n}=9\end{array}$ & $\begin{array}{c}50 \% \\
\mathrm{n}=2\end{array}$ & 0,89 \\
\hline $\begin{array}{l}\text { Efficacité } \\
\text { finale }\end{array}$ & $\mathbf{6 8 , 6 \%}$ & $\begin{array}{c}64,7 \% \\
\mathrm{n}=11\end{array}$ & $\begin{array}{c}68,7 \% \\
\mathrm{n}=11\end{array}$ & 0,81 & $\begin{array}{c}68 \% \\
\mathrm{n}=17\end{array}$ & $\begin{array}{c}70 \% \\
\mathrm{n}=7\end{array}$ & 0,99 & $\begin{array}{c}57,1 \% \\
\mathrm{n}=8\end{array}$ & $\begin{array}{c}82,3 \% \\
\mathrm{n}=14\end{array}$ & $\begin{array}{c}50 \% \\
\mathrm{n}=2\end{array}$ & 0,253 \\
\hline $\begin{array}{l}\text { Taux } \\
\text { d'extirpabilité }\end{array}$ & $\mathbf{1 0 0 \%}$ & $\mathrm{NA}$ & $\mathrm{NA}$ & & $\mathrm{NA}$ & $\mathrm{NA}$ & & $\mathrm{NA}$ & $\mathrm{NA}$ & $\mathrm{NA}$ & \\
\hline $\begin{array}{l}\text { Taux de } \\
\text { migration }\end{array}$ & $\mathbf{1 8 \%}$ & $\mathrm{NA}$ & $\mathrm{NA}$ & & $\mathrm{NA}$ & $\mathrm{NA}$ & & $\mathrm{NA}$ & $\mathrm{NA}$ & $\mathrm{NA}$ & \\
\hline $\begin{array}{l}\text { Taux de } \\
\text { mortalité }\end{array}$ & $\mathbf{1 7 , 1 \%}$ & $\begin{array}{c}23,5 \% \\
\mathrm{n}=4\end{array}$ & $\begin{array}{c}12,5 \% \\
\mathrm{n}=2\end{array}$ & 0,66 & $\begin{array}{c}24 \% \\
\mathrm{n}=6\end{array}$ & $\begin{array}{c}0 \% \\
\mathrm{n}=0\end{array}$ & 0,296 & $\begin{array}{c}28,6 \% \\
\mathrm{n}=4\end{array}$ & $\begin{array}{c}11,8 \% \\
\mathrm{n}=2\end{array}$ & $\begin{array}{c}0 \% \\
\mathrm{n}=0\end{array}$ & 0,44 \\
\hline $\begin{array}{l}\text { Taux de } \\
\text { reprise chir }\end{array}$ & $\mathbf{1 4 , 3 \%}$ & $\begin{array}{c}11,8 \% \\
\mathrm{n}=2\end{array}$ & $\begin{array}{c}18,8 \% \\
\mathrm{n}=3\end{array}$ & 0,66 & $\begin{array}{c}8 \% \\
\mathrm{n}=2\end{array}$ & $\begin{array}{c}30 \% \\
\mathrm{n}=3\end{array}$ & 0,128 & $\begin{array}{c}14,3 \% \\
\mathrm{n}=2\end{array}$ & $\begin{array}{c}5,9 \% \\
\mathrm{n}=1\end{array}$ & $\begin{array}{c}50 \% \\
\mathrm{n}=2\end{array}$ & 0,091 \\
\hline $\begin{array}{l}\text { Délai médian } \\
\text { de guérison (j) }\end{array}$ & $\mathbf{4 4}$ & 44 & 43,5 & $\mathrm{NS}$ & 42 & 52 & $\mathrm{NS}$ & 45 & 44 & 42 & $N S$ \\
\hline
\end{tabular}

\section{$\underline{\text { Tableau } 2}$}




\section{SERMENT D'HIPPOCRATE}

Au moment d'être admis(e) à exercer la médecine, je promets et je jure d'être fidèle aux lois de l'honneur et de la probité.

Mon premier souci sera de rétablir, de préserver ou de promouvoir la santé dans tous ses éléments, physiques et mentaux, individuels et sociaux.

Je respecterai toutes les personnes, leur autonomie et leur volonté, sans aucune discrimination selon leur état ou leurs convictions. J'interviendrai pour les protéger si elles sont affaiblies, vulnérables ou menacées dans leur intégrité ou leur dignité. Même sous la contrainte, je ne ferai pas usage de mes connaissances contre les lois de l'humanité.

J'informerai les patients des décisions envisagées, de leurs raisons et de leurs conséquences.

Je ne tromperai jamais leur confiance et n'exploiterai pas le pouvoir hérité des circonstances pour forcer les consciences.

Je donnerai mes soins à l'indigent et à quiconque me les demandera. Je ne me laisserai pas influencer par la soif du gain ou la recherche de la gloire.

Admis(e) dans l'intimité des personnes, je tairai les secrets qui me seront confiés. Reçu(e) à l'intérieur des maisons, je respecterai les secrets des foyers et ma conduite ne servira pas à corrompre les moeurs.

Je ferai tout pour soulager les souffrances. Je ne prolongerai pas abusivement les agonies. Je ne provoquerai jamais la mort délibérément.

Je préserverai l'indépendance nécessaire à l'accomplissement de ma mission. Je n'entreprendrai rien qui dépasse mes compétences. Je les entretiendrai et les perfectionnerai pour assurer au mieux les services qui me seront demandés.

J'apporterai mon aide à mes confrères ainsi qu'à leurs familles dans l'adversité.

Que les hommes et mes confrères m'accordent leur estime si je suis fidèle à mes promesses ; que je sois déshonoré(e) et méprisé(e) si j'y manque.

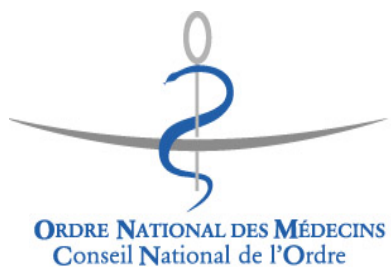





\section{RESUME}

Introduction La fistule anastomotique est l'une des principales complications de l'œsophagectomie pour cancer de l'œsophage ou de la jonction œso-cardiale. Elle serait responsable de $40 \%$ des décès en post-opératoire de cette chirurgie. Depuis 10 ans, la prise en charge endoscopique de ces fistules est devenue une alternative face à la reprise chirurgicale ou au traitement conservateur. L'objectif de cette étude est d'évaluer l'efficacité et les caractéristiques du traitement endoscopique dans cette indication.

Matériel et Méthodes Dans cette étude monocentrique, 35 patients inclus consécutivement ont été traités endoscopiquement pour une fistule anastomotique après œsophagectomie pour cancer de l'œsophage ou de la jonction œso-cardiale entre juin 2010 et juin 2014. Toutes les procédures endoscopiques ont été réalisées dans l'unité d'endoscopie de l'hôpital Nord à Marseille, par des endoscopistes interventionnels, chez des patients intubés sous anesthésie générale, avec un endoscope à large canal opérateur (3,8mm, Pentax, Japon) sous contrôle scopique. Un contrôle endoscopique était systématiquement effectué 4 à 8 semaines après afin de s'assurer de l'efficacité ou de la nécessité d'un traitement additionnel. L'efficacité primaire et secondaire, le délai entre la chirurgie, le diagnostic et l'endoscopie, le nombre de reprises endoscopiques, le matériel utilisé (prothèse, clip, drain), les complications et le taux de mortalité ont été évalués et une analyse uni et multivariée a été réalisée afin de mettre en évidence des facteurs prédictifs de succès.

Résultats Parmi les 35 patients, il y avait 4 femmes et 31 hommes avec un âge moyen de 61,7 ans \pm 8,9 [43-85]. Les principales techniques chirurgicales employées ont été celles de Lewis-Santy pour $48,6 \%$ des cas et de Akiyama pour $45,7 \%$. 71,4\% des patients avaient bénéficié d'une radiochimiothérapie néoadjuvante et $77,1 \%$ étaient hospitalisés en réanimation. Le délai médian entre la chirurgie et le premier traitement endoscopique était de 8,5 jours (IQR ; 6,00-18,25). 88,6\% des patients ont été traités par une prothèse métallique œsophagienne avec un taux final d'extirpabilité de $100 \%$ et un taux de migration de $18 \%$. Le nombre moyen d'endoscopies réalisées par patient a été de 2,6 $\pm 1,57$ [1-10] avec un nombre moyen de prothèses œsophagiennes de 1,6 $\pm 1,35$ [0-7] par patient. L'efficacité primaire du traitement endoscopique a été de $48,6 \%$ et l'efficacité finale de $68,6 \%$. Le taux de mortalité en cas de traitement endoscopique a été de $17 \%$, aucun décès n'étant lié au traitement endoscopique par lui-même. Le type de technique chirurgicale, la réalisation d'une radiochimiothérapie pré-opératoire et la taille de l'orifice fistuleux n'ont pas eu d'influence sur l'efficacité du traitement endoscopique que ce soit en analyse uni ou multivariée.

Conclusion La prise en charge endoscopique des fistules anastomotiques post-œsophagectomie est efficace, en particulier avec les prothèses œsophagiennes métalliques et n'entraine aucune complication liée au traitement. Elle est de plus associée à un taux de mortalité de 17\% inférieur à celui de la reprise chirurgicale ou du traitement conservateur (40 à 100\%) (1).

\section{Références}

1. Alanezi K, Urschel JD. Mortality secondary to esophageal anastomotic leak. Ann Thorac Cardiovasc Surg Off J Assoc Thorac Cardiovasc Surg Asia. avr 2004;10(2):71-5.

Mots clés : Fistules anastomotiques, chirurgie œsophagienne, endoscopie interventionnelle. 